\title{
New Smart Passive Graphical Islanding Detection Method
}

\author{
Noha H. El-Amary \\ Department of Electrical and Control Engineering, College of Engineering, \\ Arab Academy for Science, Technology and Maritime Transport (AASTMT), Cairo, Egypt.
}

\begin{abstract}
As there is still a great argument on islanding detection methods, this paper presents a new smart passive graphical islanding detection method. The new method depends on measuring the instantaneous variation in phase voltage angle $(\theta)$ related to the instantaneous variation in phase voltage magnitude $(V)$ and drawing the relationship between them. The traditional passive methods have performance deficiencies. On the other hand, the active methods are more accurate islanding detection methods, but perturb the electrical system. So, the need for a new detection method which can be accurate, doesn't affect the system behavior, and through which both active and passive methods disadvantages can be avoided is an essential requirement. The continuous updates in Wide Area Monitoring, Protection and Control (WAMPC) technology and Phasor measurement units (PMU) facilitate the idea of measuring and tracing the voltage angle variation. The concept of the new proposed smart passive islanding detection method depends on observing the behavior of the variation of voltage magnitude versus the voltage angle variation $(V-\theta)$ of the bus which connects the suggested island with the main power grid. During islanding, sometimes the variation of the voltage magnitude is not significant to be detected, especially in case of low power island. The variation of the voltage angle presents an accurate indication with high sensitivity to the different sorts of system transients. The angle variation behavior differs in load changing, islanding, faulted network, and normal operation conditions. The relationship between $(V-\theta)$ is confirmed by the graphical relationship between their derivatives $(d V / d t-d \theta / d t)$. $V$ versus $\theta$ and $d V / d t$ versus $d \theta / d t$ are studied and analyzed in this research to verify the concept of the new method. The new proposed smart method is applied to four different power systems having the same main power grid. The wind electrical power generation unit of the studied power systems has different generation and loading capacities in each of the four studied cases. The studied cases are classified into low, high, and medium (with two different loading status) wind electrical power generation capacity. For each studied case, the system normal operation, load disconnection, islanding, and three phase fault conditions are discussed. In the four studied cases, the results verify the completely different graphical behaviors for each transient condition. So, the concept of the new method is verified.
\end{abstract}

Keywords: Smart passive islanding detection, voltage phase versus voltage magnitude variation $(d V-d \theta)$ detection, Wide Area Monitoring, Protection and Control (WAMPC), voltage phase versus voltage magnitude $(V-\theta)$ detection, Phasor Measurement Unit $(P M U)$.

\section{INTRODUCTION}

High stability and precious power system monitoring, case detection, protection, and control are essential requirements for any power network. Islanding detection is one of the issues that is still considered an open research field which needs extra work.

Intentional and unintentional islanding are the two modes of the islanding process [1]-[6]. The intentional islanding is planned to divide a part of the power network into islands (micro-grids) in case of any abnormal disturbance occurrence or maintenance purposes. The separated island is constructed from Distributed Generators (DGs) which supply the island local loads through high performance and well-planned synchronized manner. The intentional islanding can be considered as an acceptable islanding mode, since it saves the power system continuity with satisfied performance [1].

The unintentional islanding is an unplanned islanding mode. It can affect the power system in a random violent way, due to synchronization loss of the grid. The island's DGs almost loss their voltage and frequency references, which may lead to the damage of the loads and system equipments in the separated island and hazardous situations [1]-[6]. The islanding detection is a vital task. A fast, accurate, and economic method that doesn't affect the system stability should be targeted.

The existed detection methods are classified into three main categories in addition to the hybrid the methods [1]-[6]. The main categories are: a- passive methods, b- active methods, and c- Remote (communication base) methods. The passive and active methods are also classified as local techniques [2]. The hybrid methods are combinations of the main methods. Each islanding detection method has its own advantages and disadvantages.

The passive islanding detection techniques depend on; a- voltage detection (under and over voltage detection), $b$ - frequency detection (under and over frequency detection, and rate of change of frequency detection) 
c- phase detection (voltage phase jump detection), d- power detection (rate of change of output power), or eharmonic distortion detection (voltage and current harmonics detection). Although the passive methods don't affect the power system status and stability behavior, they have a defective detection characteristics with obvious Non Detection Zone (NDZ).

The active islanding detection techniques reply on; a- voltage perturbations techniques (Sandia Voltage Shift (SVS)), b- frequency perturbations techniques (Sandia Frequency Shift (SFS), Slip Mode Frequency Shift (SMFS), Active Frequency Drift (AFD), and Frequency Jump (FJ)), c- detection of impedance at specific frequency, d- variation of active power and reactive power (reactive power export error detection), or e- positive feedback technique. The active islanding detection techniques are more accurate than the passive techniques but they may affect the stability of the power system negatively.

The remote islanding detection techniques consist of; a- communication based techniques (power line signaling scheme, transfer trip scheme, and Power Line Carrier Communication (PLCC)), or b- impedance insertion. The remote detection techniques are the most accurate islanding detection methods, however they are expensive [1]-[6].

So the need for a new islanding detection method, which has the advantages of all the previous mentioned techniques and avoids their disadvantage, is an essential aim. The continuous development and improvement in the Wide Area Monitoring, Protection, and Control (WAMPC) technology helps in finding a new islanding detection methods. Also, WAMPC technology can be considered not so expensive, due to its great rate of spreading. The Phasor Measurement Unit (PMU) is one of the main elements of the WAMPC system. It can revolutionize the way of power systems monitoring and control [7]. PMU is an electronic device which uses state-of-the-art digital signal processors that can measure $50 / 60 \mathrm{~Hz}$ AC waveforms (voltages and currents) typically at a rate of 48 samples per cycle (2880 samples per second). The analog AC waveforms are digitized by an Analog to Digital converter for each phase. Digital signal processing techniques are used to compute the voltage and current phasors. PMUs come in different sizes. Some of the larger ones can measure up to 10 phasors plus frequency while others only measure from one to three phasors plus frequency [8], [9].

In this paper, PMU and WAMPC technology are utilized in monitoring the voltage magnitude and phase of the bus, which connects the suggested island with the grid. The measured values are used in the new smart graphical passive islanding detection method.

\section{ELECTRICAL POWER SYSTEM ISLANDING DETECTION}

The electrical system power flow is affected by the system topology, generation, and loads. It affects the values of buses phasor voltages (magnitudes and angles) as known from power flow studies equations (1) - (4). A simple two buses power system that consists of generation, transmission, and load units are presented in Fig. 1.

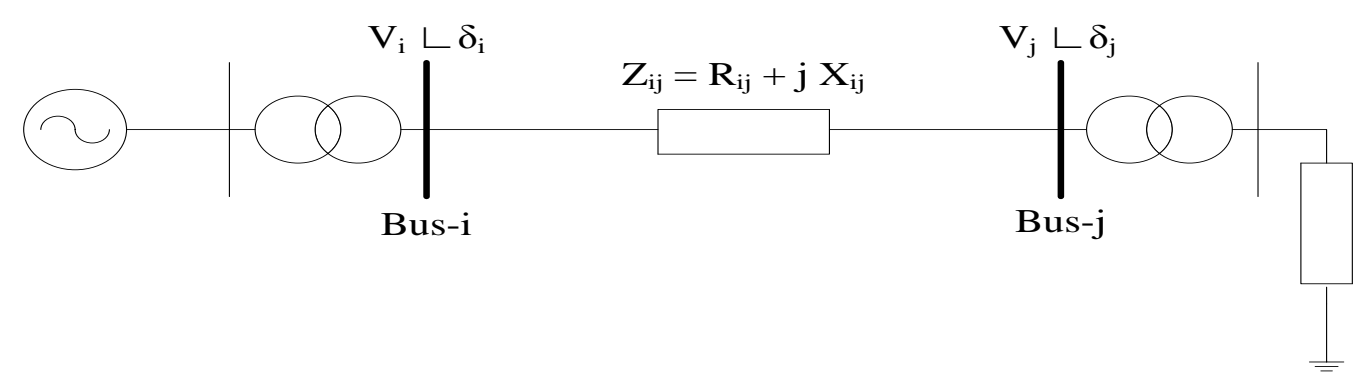

Fig. 1. A simple two buses power system

$$
\begin{aligned}
& \mathrm{P}_{\mathrm{i}}=\Sigma\left(\mathrm{V}_{\mathrm{i}} \mathrm{V}_{\mathrm{j}} \mathrm{Y}_{\mathrm{ij}} \cos \left(\delta_{\mathrm{i}}-\delta_{\mathrm{j}}+\theta_{\mathrm{ij}}\right)\right), \quad \text { for } \mathrm{j}=1,2, \ldots, \mathrm{N} \\
& \mathrm{Q}_{\mathrm{i}}=\Sigma\left(\mathrm{V}_{\mathrm{i}} \mathrm{V}_{\mathrm{i}} \mathrm{Y}_{\mathrm{ij}} \sin \left(\delta_{\mathrm{i}}-\delta_{\mathrm{j}}+\theta_{\mathrm{ij}}\right)\right), \quad \text { for } \mathrm{j}=1,2, \ldots, \mathrm{N} \\
& \mathrm{P}_{\mathrm{ij}}=\mathrm{V}_{\mathrm{i}} \mathrm{V}_{\mathrm{i}} \mathrm{Y}_{\mathrm{ij}} \cos \left(\delta_{\mathrm{i}}-\delta_{\mathrm{i}}+\theta_{\mathrm{ij}}\right)-\mathrm{V}_{\mathrm{i}}^{2} \mathrm{Y}_{\mathrm{ij}} \cos \left(\theta_{\mathrm{ij}}\right) \\
& \mathrm{Q}_{\mathrm{ij}}=\mathrm{V}_{\mathrm{i}} \mathrm{V}_{\mathrm{j}} \mathrm{Y}_{\mathrm{ij}} \sin \left(\delta_{\mathrm{i}}-\delta_{\mathrm{j}}+\theta_{\mathrm{ij}}\right)-\mathrm{V}_{\mathrm{i}}{ }^{2}\left(\mathrm{Y}_{\mathrm{ij}} \sin \left(\theta_{\mathrm{ij}}\right)+\mathrm{B}_{\text {capij }}\right)
\end{aligned}
$$

where;

$$
\begin{array}{cl}
\mathrm{Y}_{\mathrm{ij}} & : \text { is the admittance magnitude of the line connected bus-i and bus } \mathrm{j} . \\
\theta_{\mathrm{ij}} & : \text { is the admittance angle of the line connected bus } \mathrm{i} \text { and bus } \mathrm{j} . \\
\delta_{\mathrm{i}} & : \text { is the angle of the bus } \mathrm{i} \text { voltage. } \\
\mathrm{B}_{\text {capij }} & : \text { is the total line charging susceptance. } \\
\mathrm{N} & : \text { is the total number of the network buses. }
\end{array}
$$


In case of any power system disturbance, both voltage magnitude and angle of the system buses are affected with different percentages. The voltage angle is more sensitive to the power system disturbance. It can be considered as a good mirror to the system stability status and behavior. As the system subjected to a load variation, the bus voltage angle changes softly, saving its reference (the main voltage reference of the power network). On the other hand, if the system is subjected to an unintentional islanding, the bus voltage angle losses its reference and starts to oscillate searching for a new reference. During islanding, the bus voltage magnitude may have slight variations without obvious changes.

\section{STUDIED SYSTEMS SIMULATIONS AND RESULTS}

The proposed study of the smart passive islanding detection method is applied to four simulated power system cases with different wind electrical generation capacities. The four studied cases are designed and simulated using the Matlab/Simulink program. There is a main grid connected to the wind electrical power generation unit in each studied case. The main grid is similar in the four studied cases. It consists of an equivalent large capacity power supply with its complementary system, and a medium length transmission line as shown in Fig. 2. The main grid is connected to the wind electrical energy generation subsystem through an ideal Switch Gear (SG). The wind electrical energy generation subsystem has the same construction in the first three studied cases, although it has different generation and load power capacities. The wind electrical energy generation subsystem is designed of a wind generation unit which is connected to a dynamic load parallel to a parallel R-L-C load through an ideal SG as illustrated in Fig. 3. Figure 4 presents the wind turbine with an induction generator connected to a STATCOM, which are the main components of the wind generation unit.

In the fourth studied case, the wind electrical power generation unit consists of four wind generation subsystems which are connected to two parallel loads (a dynamic load and a parallel R-L-C load) as presented in Fig. 5. The wind construction of the four generation units are similar to that of the other three studied case.

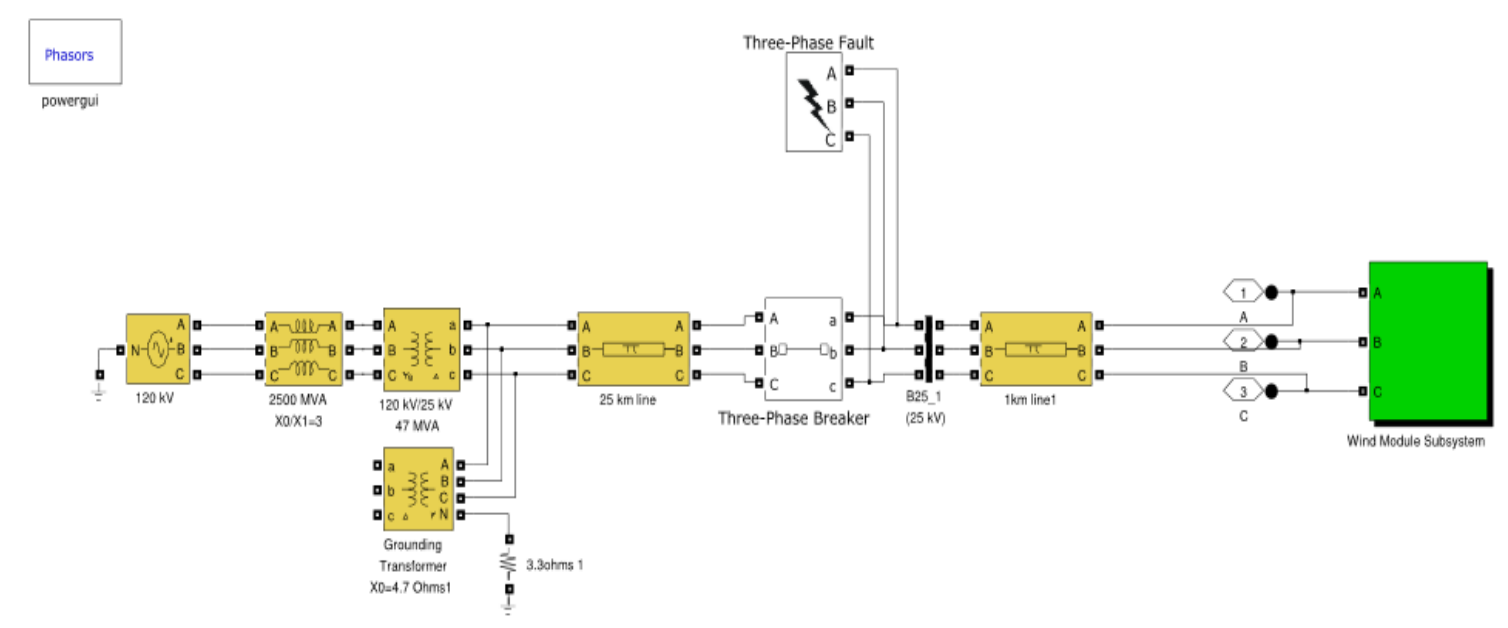

Fig. 2. The Matlab/Simulink simulated studied power system

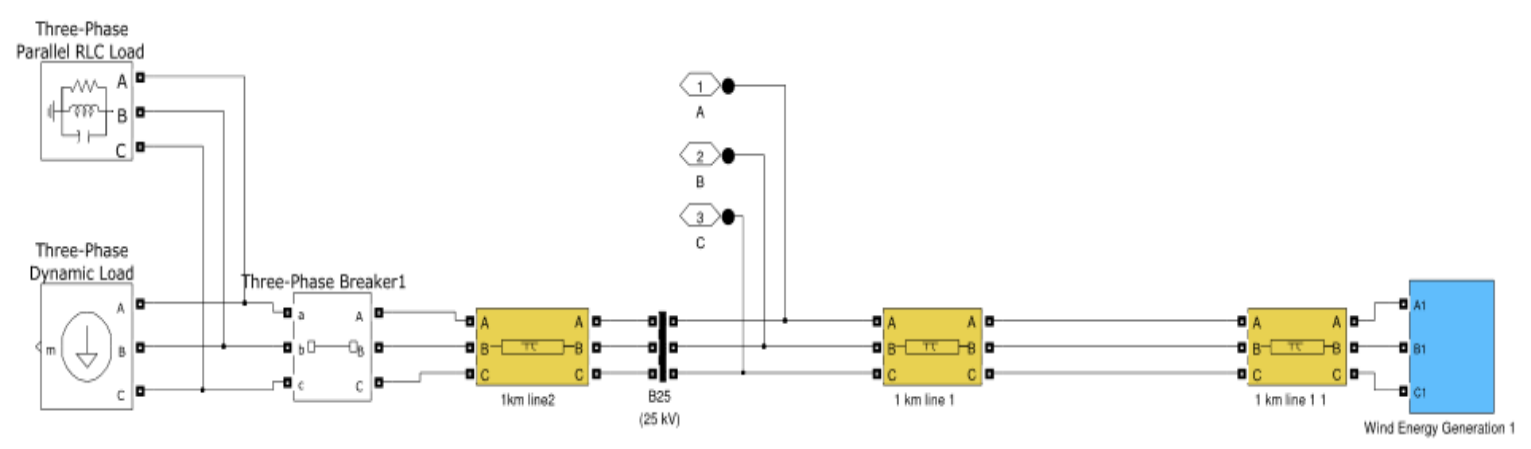

Fig. 3. The wind electrical power generation unit 


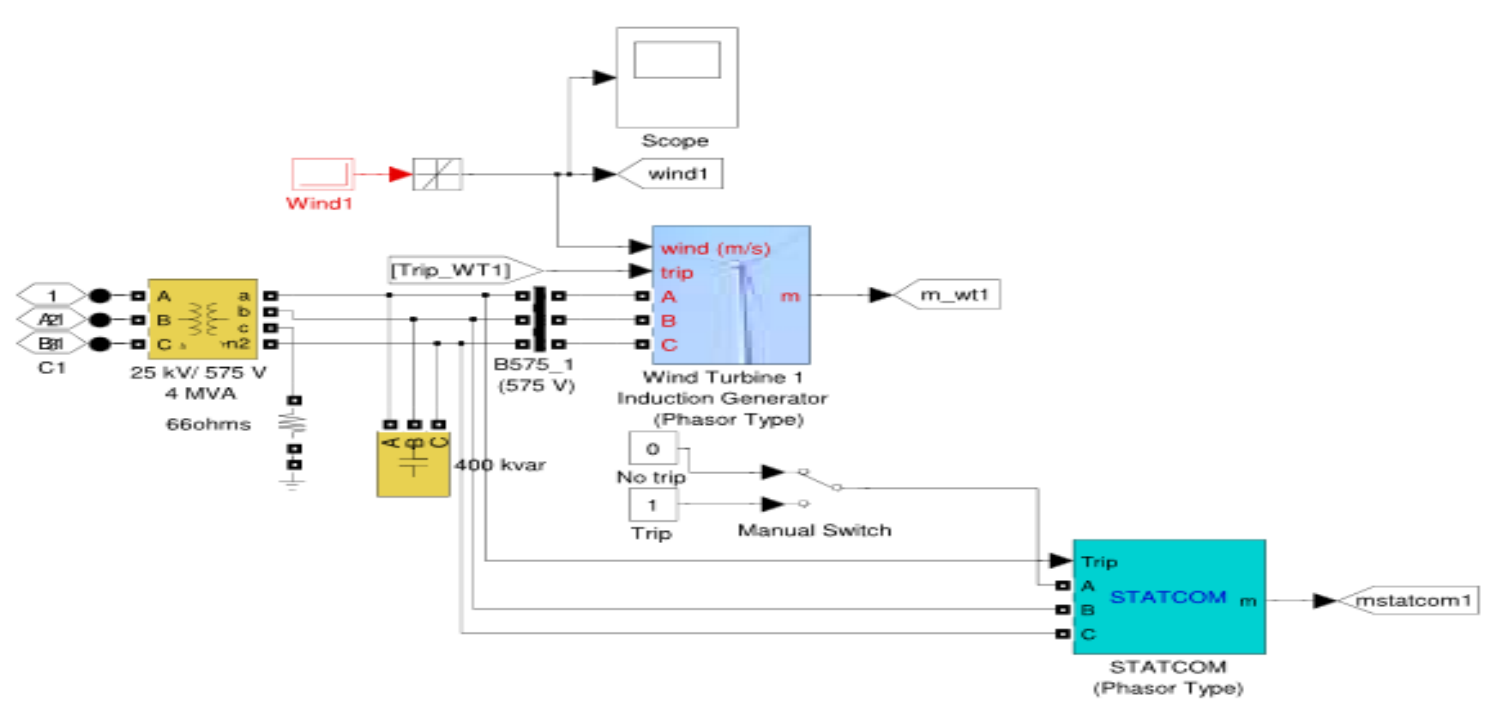

Fig. 4. The wind turbine with the induction generator and its supplementary system

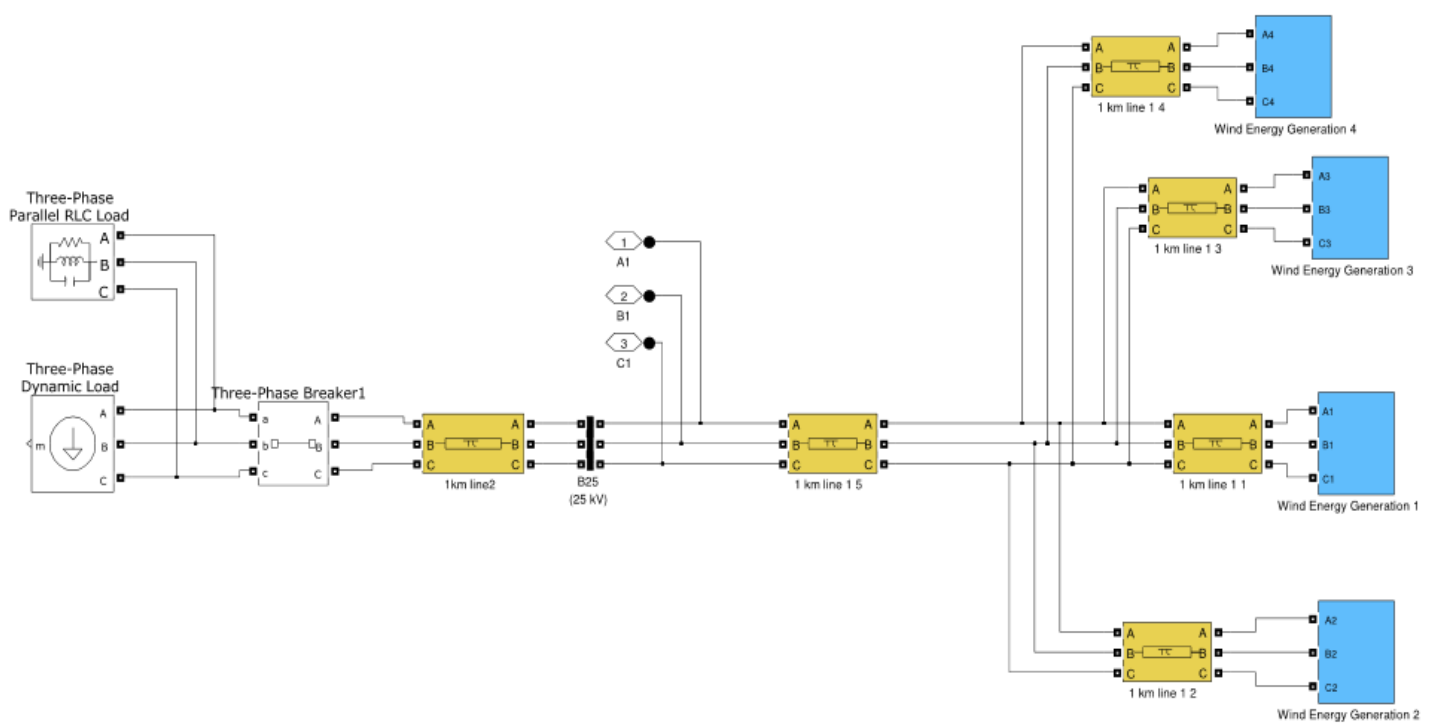

Fig. 5. The wind electrical power generation unit of the fourth studied case (DG with large power generation

\section{The Studied Cases:}

capacity)

For each study case, the system output phasor voltage is observed on the bus connected the suggested island with the main grid (shown in Fig. 1). It is observed for the system normal operation, load disconnection, islanding, and three phase fault statuses. The variation of voltage magnitude (in pu) and angle (in degrees) with time (in sec.), and the graphical presentation of voltage magnitude versus voltage angle (V - $\theta$ ) are studied for each system status. The voltage magnitude and angle differentiations(dV/dt and $\mathrm{d} \theta / \mathrm{dt}$ respectively) and their variation with time are also clarified. The graphical behavior of the voltage magnitude derivative versus the voltage angle derivative $(\mathrm{dV} / \mathrm{dt}-\mathrm{d} \theta / \mathrm{dt})$ is discussed for each case.

All system disturbances are applied to the power network at time $\mathrm{t}=8 \mathrm{sec}$., after the system completely reaches its steady state stability. These disturbances can be classified into a- load disconnection, $b$ - islanding case by disconnecting the main grid, and c- three phase fault status.

\section{First Case:}

\section{Medium Power Capacity Wind Electrical Power Generation Unit with Load-A}

In this case, the power capacity of the wind electrical power generation unit is $3 \mathrm{MVA}$. It is connected to load-A with a dynamic load of $10 \mathrm{~kW}$ and $3 \mathrm{kVAR}$ which is parallel to a static (parallel R-L-C) load of $2.5 \mathrm{MW}$, $0.1 \mathrm{kVAR}$ inductive and $0.1 \mathrm{kVAR}$ capacitive. 


\section{A. Normal Operation Status:}

For normal operation condition, the voltage magnitude $(\mathrm{V}$ in pu) and angle ( $\theta$ in degrees) waveforms versus time (in sec.), of the bus which connects the suggested island with the grid, are illustrated in Fig. 6. The graphical smart islanding detection method $(\mathrm{V}$ versus $\theta)$ is presented in Fig. 7. The $(\mathrm{V}-\theta)$ shows a bi-directional spiral variation behavior around one nucleus with a final steady state point at $\mathrm{V}=0.99 \mathrm{pu}$ and $\theta=-27.6^{\circ}$. The time waveforms of voltage magnitude and angle derivatives (dV/dt and $\mathrm{d} \theta / \mathrm{dt}$ respectively) are presented in Fig. 8. Figure 9 clarifies $(\mathrm{dV} / \mathrm{dt}-\mathrm{d} \theta / \mathrm{dt})$ graph. It is a closed continuous spiral shape, which starts and ends at $(0,0)$ point.
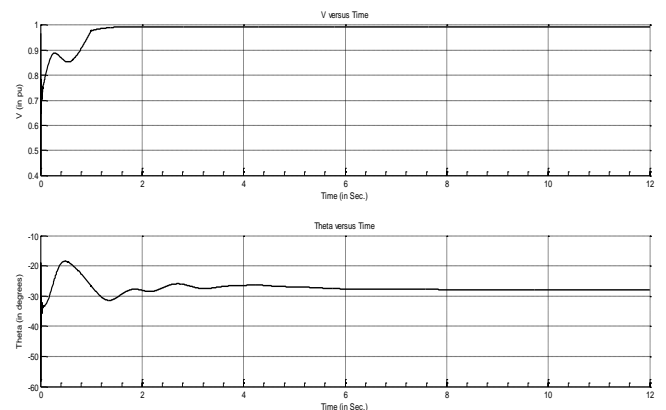

Fig. 6. The island connected bus voltage magnitude ( $\mathrm{V}$ in $\mathrm{pu})$ and angle ( $\theta$ in degrees) versus time (in sec.) of the 3MVA wind generation unit with load-A for normal operation.
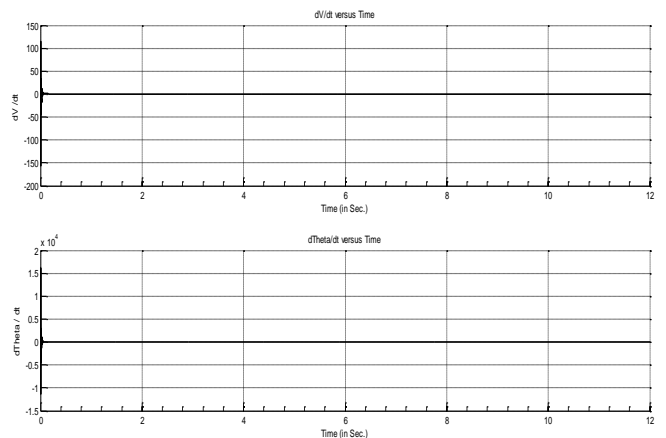

Fig. 8. The island connected bus voltage magnitude and angle derivatives ( $\mathrm{dV} / \mathrm{dt}$ and $\mathrm{d} \theta / \mathrm{dt}$ respectively) versus time of the $3 \mathrm{MVA}$ wind generation unit with load-A for normal operation.

B. Load Disconnection Status:

For the load disconnection condition, (V - Time) and ( $\theta$ - Time) are shown in Fig. 10, while (dV/dt Time) and ( $\mathrm{d} \theta / \mathrm{dt}-\mathrm{Time})$ are given in Fig. 12. The load is disconnected at $\mathrm{t}=8 \mathrm{sec}$. The bi-directional spiral (V $\theta$ ) form is illustrated in Fig. 11. It has two shifted spiral shapes with two nearby centers (nuclei). The steady state point is shifted to $\mathrm{V} \approx 1 \mathrm{pu}$ and $\theta \approx-19^{\circ}$, after load disconnection. A very small spiral shape, at the starting edge of a long sharp spike, describes the $(d V / d t-d \theta / d t)$ graph which is shown in Fig. 13. The long spike is a positive slope line based on the spiral shape.

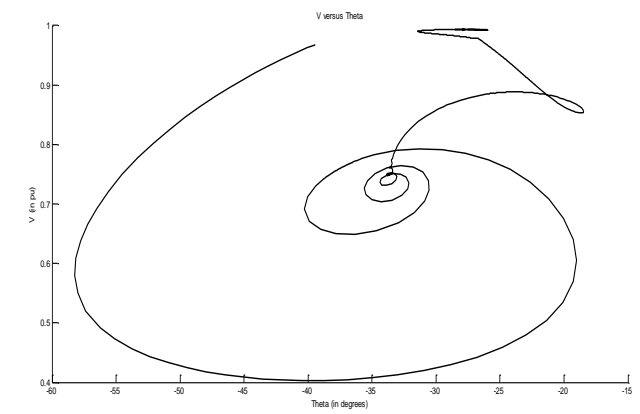

Fig. 7. The island connected bus voltage magnitude $(\mathrm{V})$ versus bus voltage angle $(\theta)$ of the 3 MVA wind generation unit with load-A for normal operation.

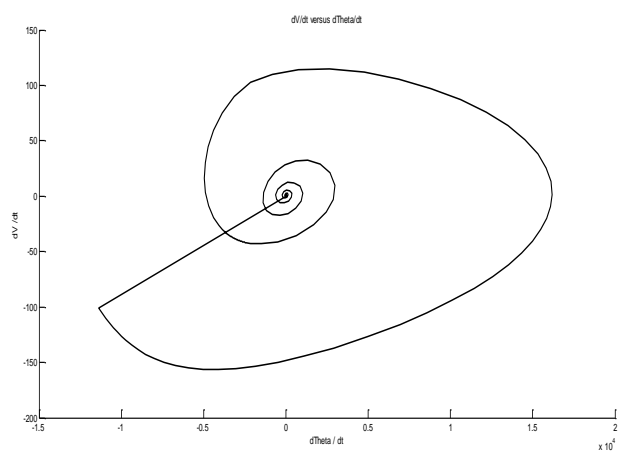

Fig. 9. The island connected bus voltage derivative $(\mathrm{dV} / \mathrm{dt})$ versus bus voltage angle derivative $(d \theta / d t)$ of the 3 MVA wind generation unit with load-A for normal operation. 

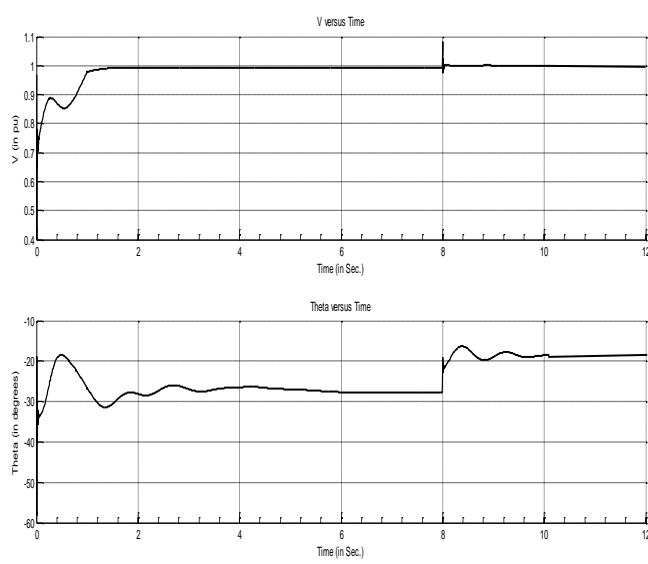

Fig. 10. V and $\theta$ versus time of the 3MVA wind generation unit with load-A for load disconnection status.
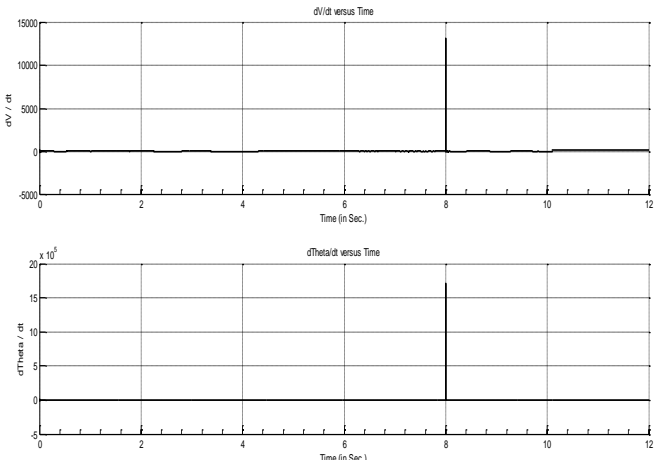

Fig. 12. $d V / d t$ and $d \theta / d t$ versus time of the $3 \mathrm{MVA}$ wind generation unit with load-A for load disconnection status.

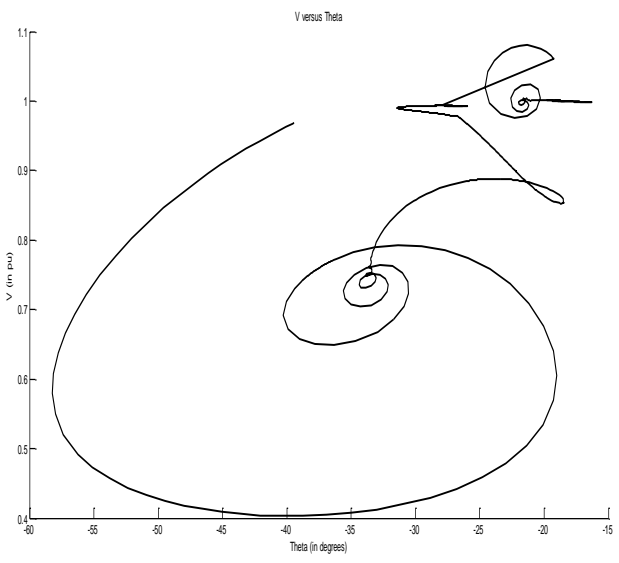

Fig. 11. V versus $\theta$ of the 3 MVA wind generation unit with load-A for load disconnection status.

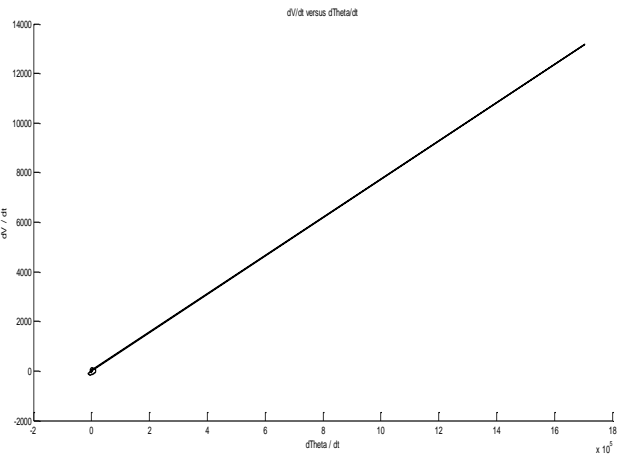

Fig. 13. $d V / d t$ versus $d \theta / d t$ of the 3 MVA wind generation unit with load-A for load disconnection status.

\section{Islanding Status:}

The time waveforms of $(\mathrm{V})$ and $(\theta)$, of the islanding condition, are presented in Fig. 14. The (dV/dt) and $(\mathrm{d} \theta / \mathrm{dt})$ time responses are illustrated in Fig. 16. The circuit breaker between the suggested island and the main grid is opened at $t=8 \mathrm{sec}$. Figure 15 clarifies the hyper oscillatory response of the suggested island after the main grid disconnection, with an instantaneous voltage rise at the time of disconnection. The relationship between $\mathrm{dV} / \mathrm{dt}$ and $\mathrm{d} \theta / \mathrm{dt}$ is shown in Fig. 17. It starts with a small spiral shape around $(0,0)$ point, then rises in form of a bi-directional positive slope sharp spike. The bi-directional spike returns to the starting point, and continues parallel to the $\mathrm{x}$-axis of the graph at $\mathrm{dV} / \mathrm{dt}=0$.
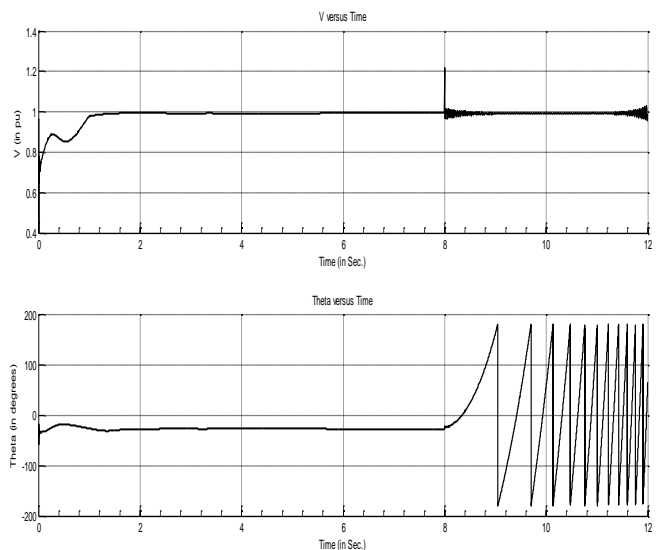

Fig. 14. V and $\theta$ versus time of the 3MVA wind generation unit with load-A for islanding status.

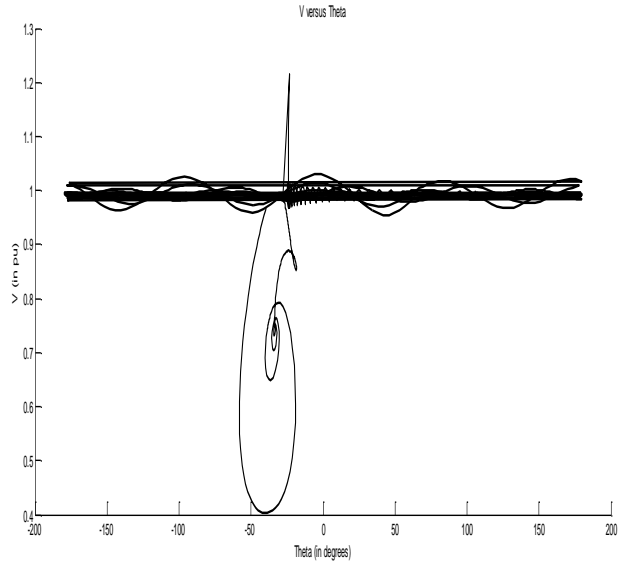

Fig. 15. V versus $\theta$ of the 3 MVA wind generation unit with load-A for islanding status. 

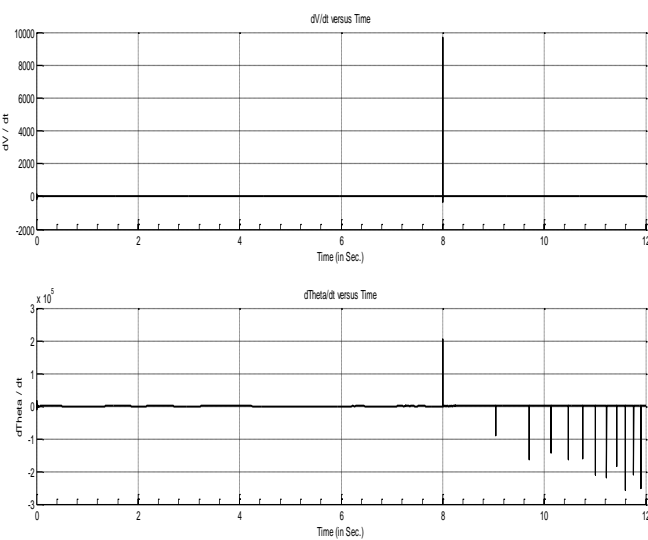

Fig. 16. $d V / d t$ and $d \theta / d t$ versus time of the $3 \mathrm{MVA}$ wind generation unit with load-A for islanding status.

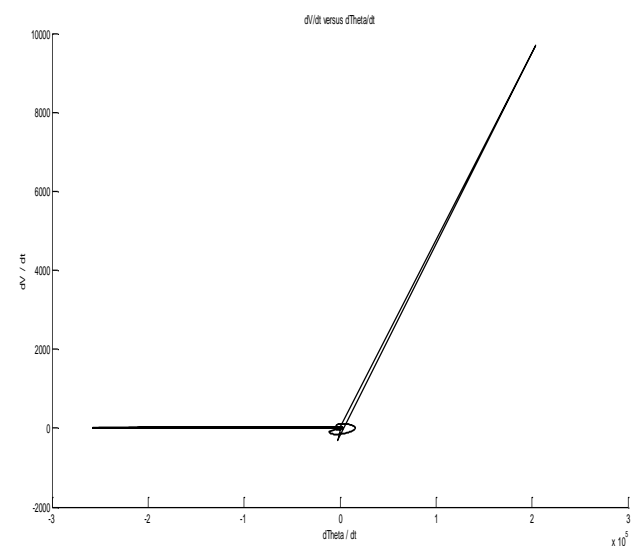

Fig. 17. $d V / d t$ versus $d \theta / d t$ of the 3 MVA wind generation unit with load-A for islanding status.

\section{Three Phase Fault Status:}

The time responses of $(\mathrm{V})$ and $(\theta)$ and their differentiations $(\mathrm{dV} / \mathrm{dt})$ and $(\mathrm{d} \theta / \mathrm{dt})$, of the three phase fault condition, are shown in Fig. 18 and Fig. 20 respectively. At $t=8$ sec., the three phase fault occurs. After fault occurrence, it is obvious that the voltage magnitude (V) drops to oscillate around V $=0$ as clarified in Fig. 19. Figure 21 illustrates $(\mathrm{dV} / \mathrm{dt})$ versus $(\mathrm{d} \theta / \mathrm{dt})$ relationship. It is constructed of an oscillatory straight line at $\mathrm{dV} / \mathrm{dt}=$ 0 , with a negative slope spike which comes from its center.
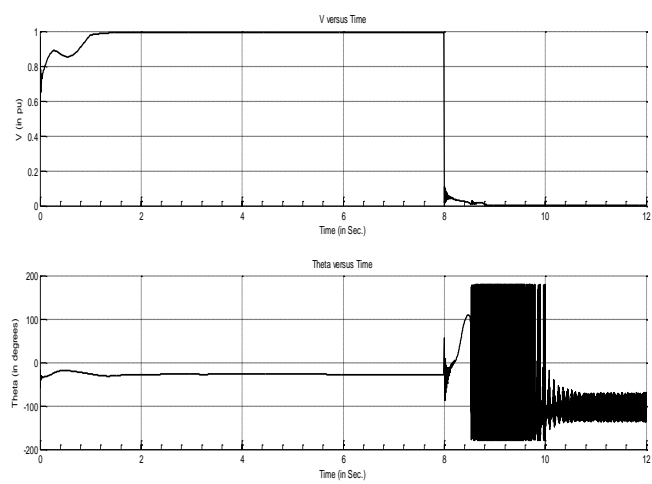

Fig. 18. $\mathrm{V}$ and $\theta$ versus time of the $3 \mathrm{MVA}$ wind generation unit with load-A for three phase fault status.
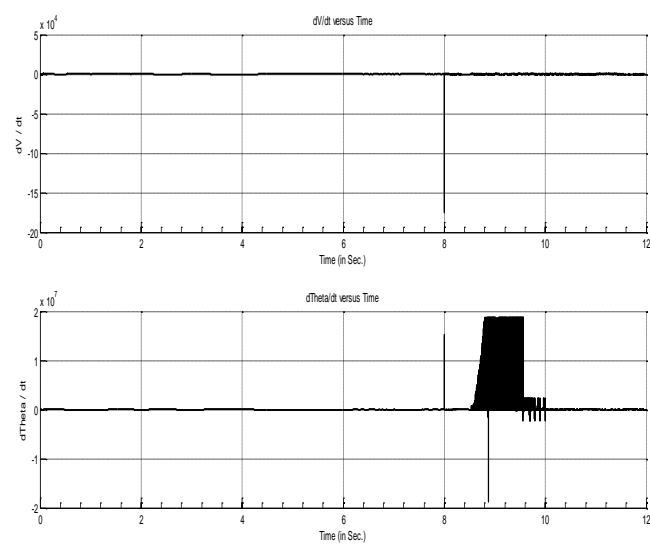

Fig. 20. $d V / d t$ and $d \theta / d t$ versus time of the $3 \mathrm{MVA}$ wind generation unit with load-A for three phase fault status.

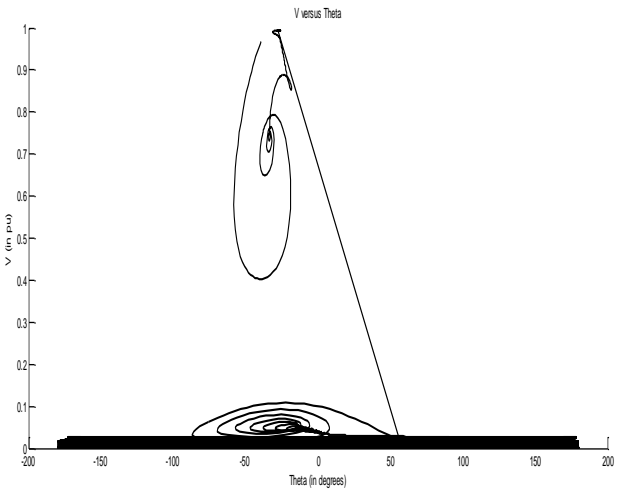

Fig. 19. V versus $\theta$ of the 3 MVA wind generation unit with load-A for three phase fault status.

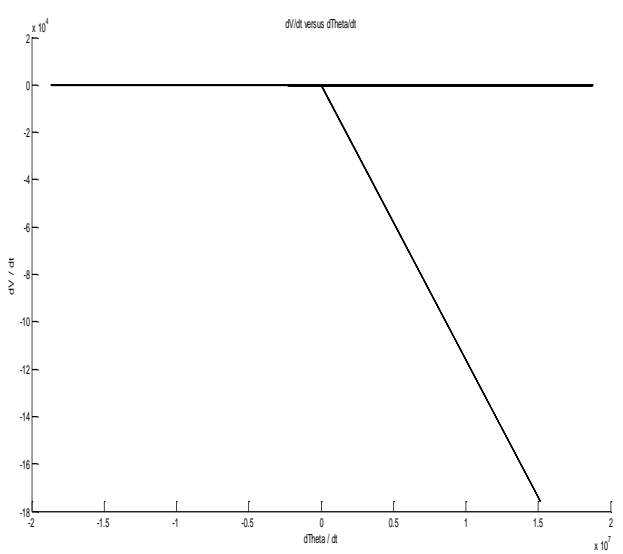

Fig. 21. $d V / d t$ versus $d \theta / d t$ of the 3 MVA wind generation unit with load-A for three phase fault status. 


\section{Second Case:}

\section{Medium Power Capacity Wind Electrical Power Generation Unit with Load-B}

The power capacity of the wind electrical power generation unit in this case is also 3 MVA. It is connected to load-B with a dynamic load of 1.3 MW and 0.3 MVAR and a parallel static (parallel R-L-C) load of 1.5 MW, $0.1 \mathrm{kVAR}$ inductive and $0.1 \mathrm{kVAR}$ capacitive.

\section{A. Normal Operation Status:}

The graphical results, for normal operation status, are shown in Fig. 22 to Fig. 25. They indicate same variation behaviors as the last studied system. In this case, the system rests at a final steady state point of $\mathrm{V} \approx 1 \mathrm{pu}$ and $\theta=-28.6^{\circ}$.

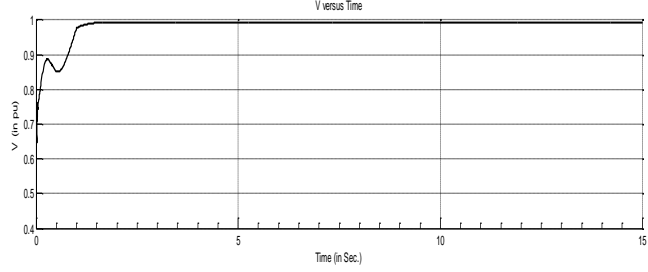

Thataesesis Tre

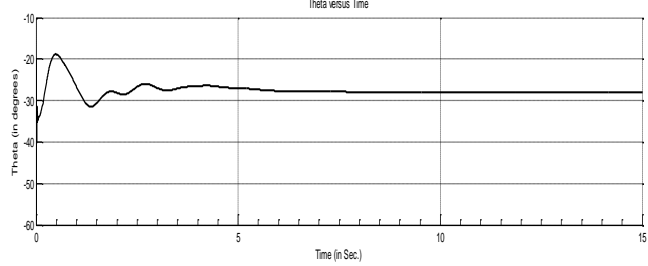

Fig. 22. V and $\theta$ versus time of the 3MVA wind generation unit with load-B for normal operation status.
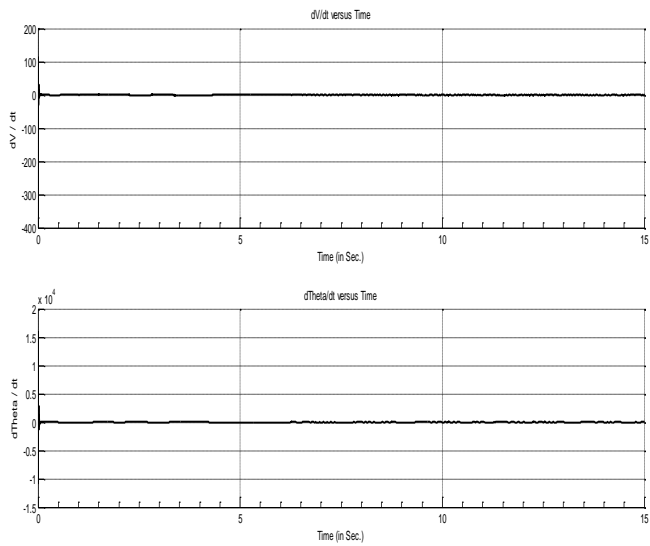

Fig. 24. $d V / d t$ and $d \theta / d t$ versus time of the 3 MVA wind generation unit with load-B for normal operation status.

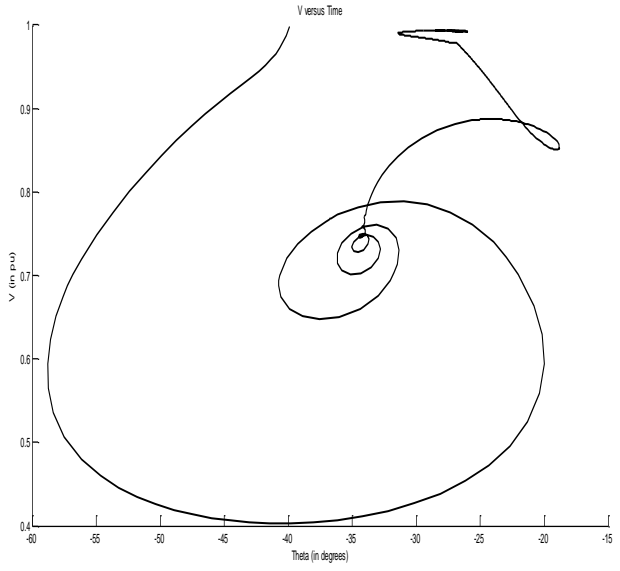

Fig. 23. V versus $\theta$ of the 3 MVA wind generation unit with load-B for normal operation status.

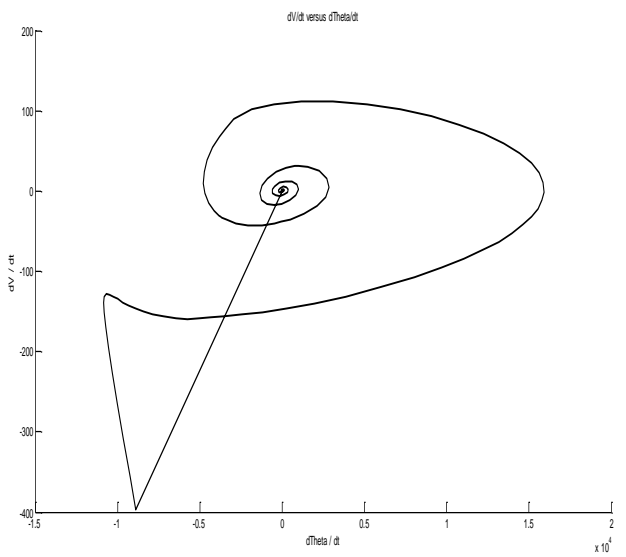

Fig. 25. $d V / d t$ versus $d \theta / d t$ of the 3 MVA wind generation unit with load-B for normal operation status.

The graphical results of the studied power system, for load disconnection, islanding, and three phase fault conditions are presented in Fig. 26 to Fig. 37. The figures show output response behaviors which are similar to that of the previous studied power system. 


\section{B. Load Disconnection Status:}

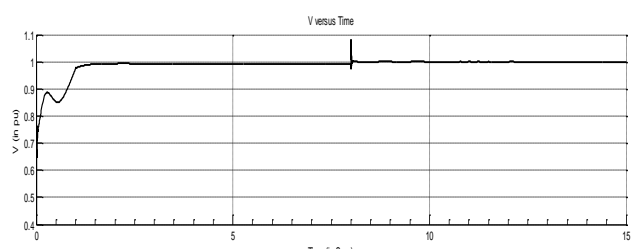

Time lin Set

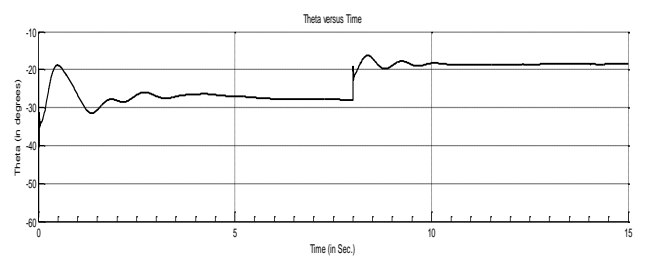

Fig. 26. $\mathrm{V}$ and $\theta$ versus time of the 3MVA wind generation unit with load-B for load disconnection status.
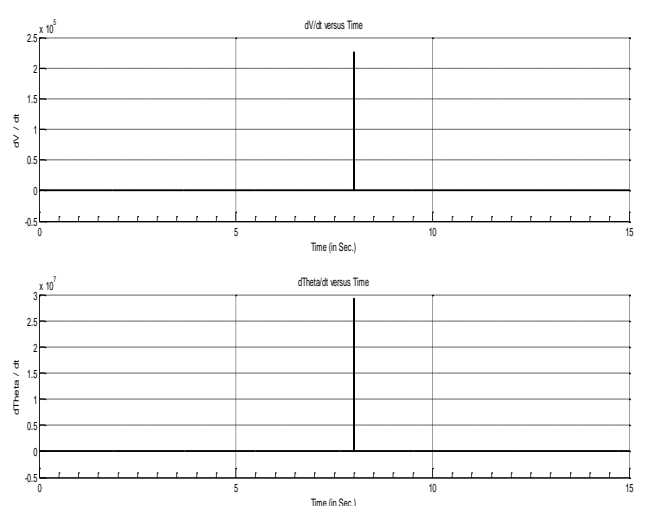

Inne insterel

Fig. 28. $d V / d t$ and $d \theta / d t$ versus time of the $3 \mathrm{MVA}$ wind generation unit with load-B for load disconnection status.

\section{Islanding Status:}
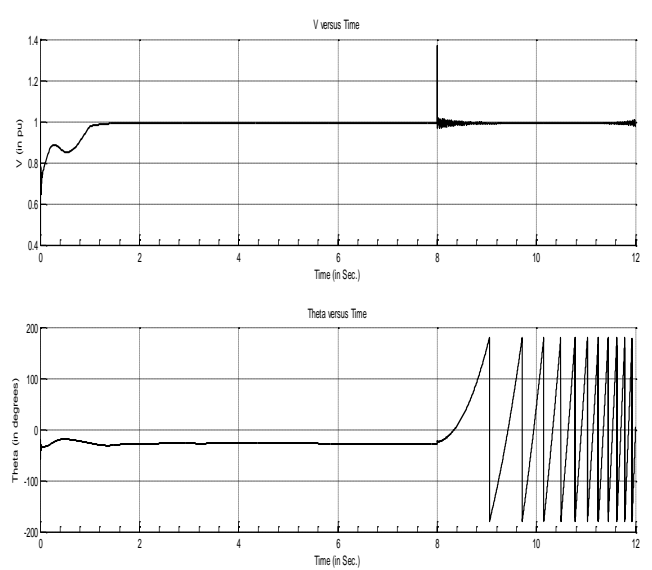

Fig. 30. $\mathrm{V}$ and $\theta$ versus time of the 3MVA wind generation unit with load-B for islanding status.

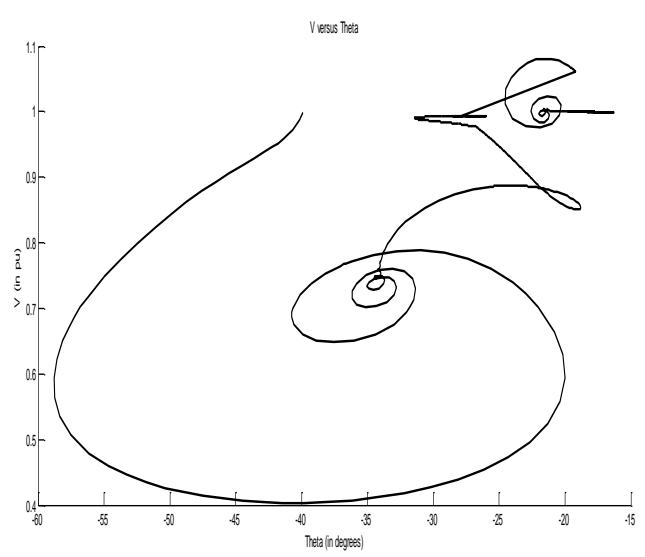

Fig. 27. V versus $\theta$ of the 3 MVA wind generation unit with load-B for load disconnection status.

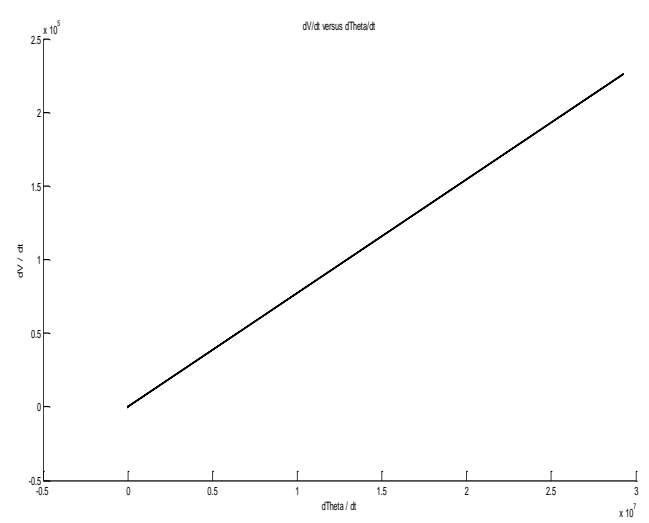

Fig. 29. $d V / d t$ versus $d \theta / d t$ of the 3 MVA wind generation unit with load-B for load disconnection status.

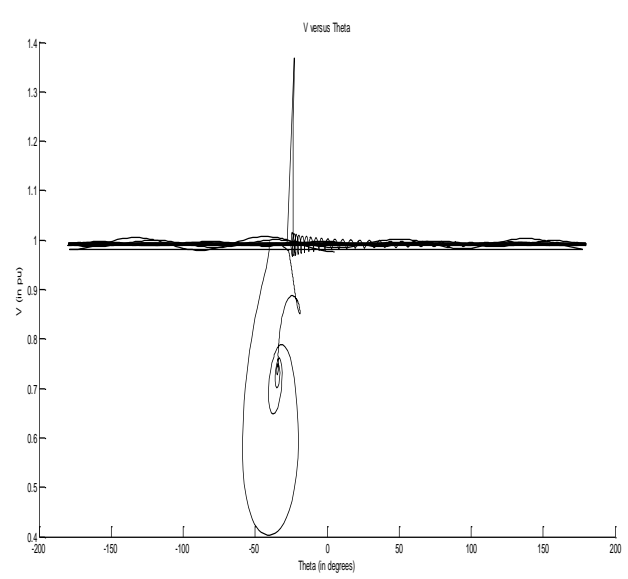

Fig. 31. V versus $\theta$ of the 3 MVA wind generation unit with load-B for islanding status. 

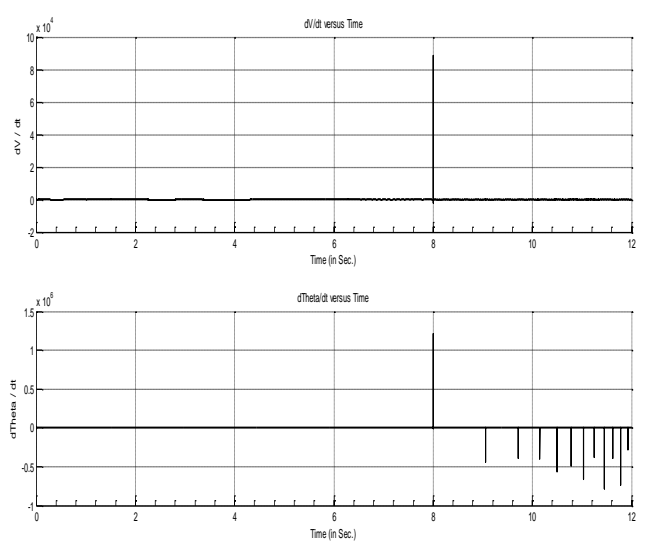

Fig. 32. $d V / d t$ and $d \theta / d t$ versus time of the $3 M V A$ wind generation unit with load-B for islanding status.

D. Three Phase Fault Status:
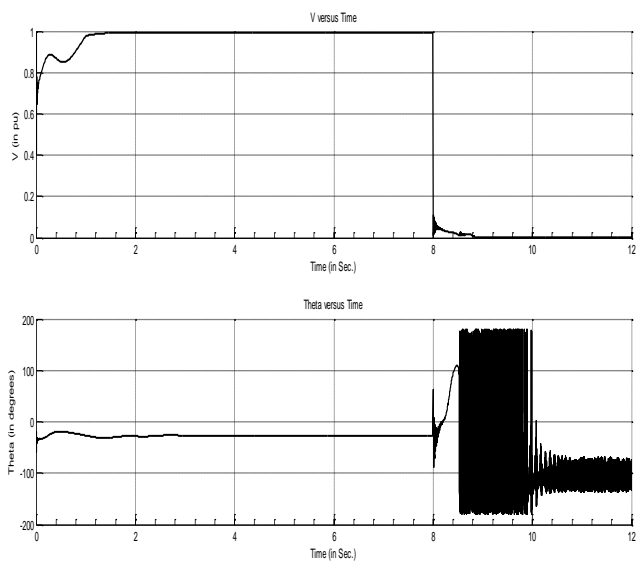

Fig. 34. V and $\theta$ versus time of the 3MVA wind generation unit with load-B for three phase fault status.
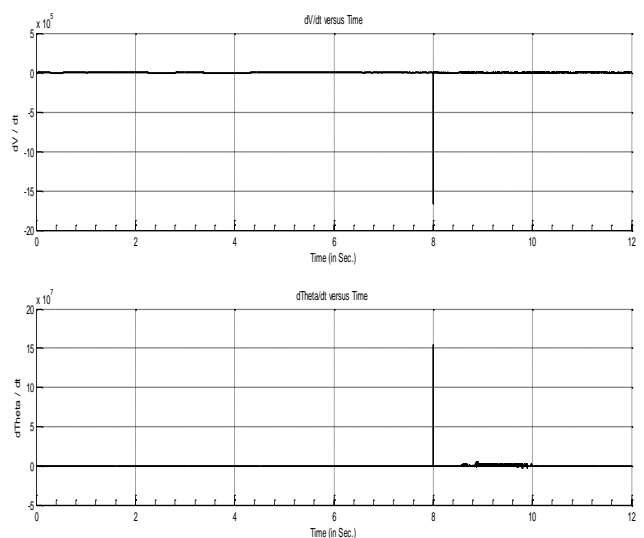

Fig. 36. $d V / d t$ and $d \theta / d t$ versus time of the $3 \mathrm{MVA}$ wind generation unit with load-B for three phase fault status.

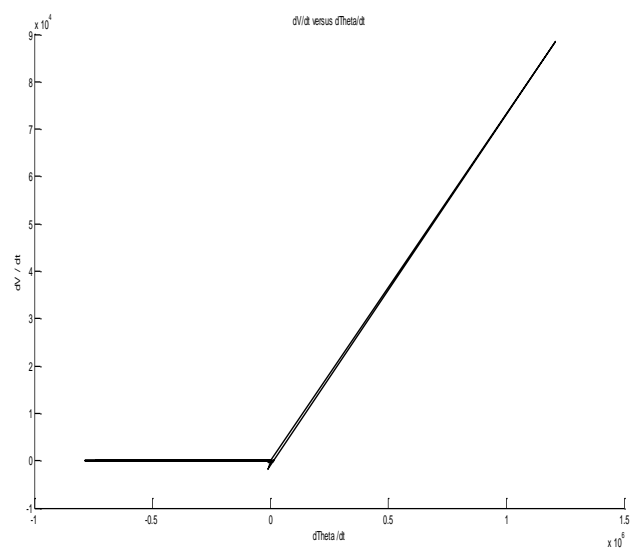

Fig. 33. $d V / d t$ versus $d \theta / d t$ of the 3 MVA wind generation unit with load-B for islanding status.

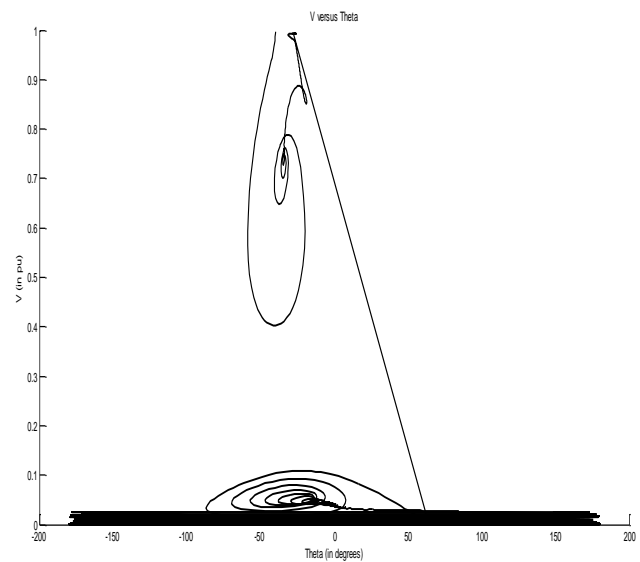

Fig. 35. V versus $\theta$ of the 3 MVA wind generation unit with load-B for three phase fault status.

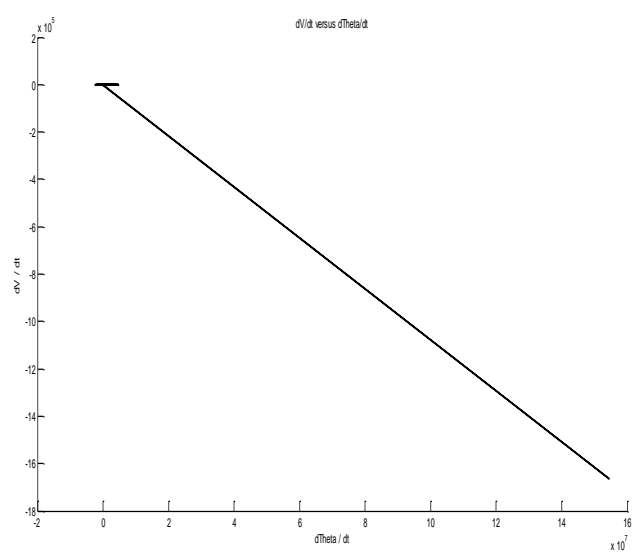

Fig. 37. $d V / d t$ versus $d \theta / d t$ of the 3 MVA wind generation unit with load-B for three phase fault status. 


\section{Third Case:}

\section{Low Power Capacity Wind Electrical Power Generation Unit}

In this case, the power capacity of the wind electrical power generation unit is $30 \mathrm{kVA}$. It is connected to a dynamic load of $10 \mathrm{~kW}$ and 30 VAR which is parallel to a static (parallel R-L-C) load of $18 \mathrm{~kW}, 0.1 \mathrm{kVAR}$ inductive and $0.1 \mathrm{kVAR}$ capacitive.

In this study case, the system responses differ from that of the medium and high power capacity wind electrical power generation unit. These differences are illustrated in Fig. 38 to Fig. 53.

\section{A. Normal Operation Status:}

The normal operation status shows a second order under-damped time response with high frequency transient oscillations for $\mathrm{V}$ and $\theta$, as seen in Fig. 38. These oscillations are reflected on the loaded spiral $(\mathrm{V}-\theta)$ and $(\mathrm{dV} / \mathrm{dt}-\mathrm{d} \theta / \mathrm{dt})$ forms, which are clarified in Fig. 39 and Fig. 41.
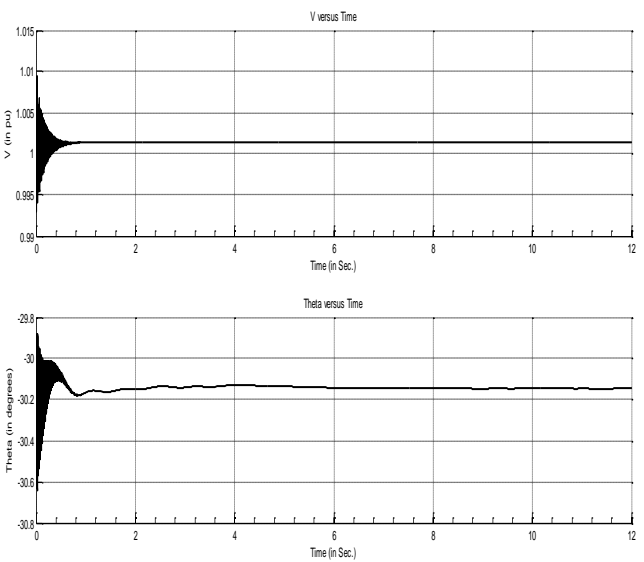

Fig. 38. $\mathrm{V}$ and $\theta$ versus time of the $30 \mathrm{kVA}$ wind generation unit for normal operation status.
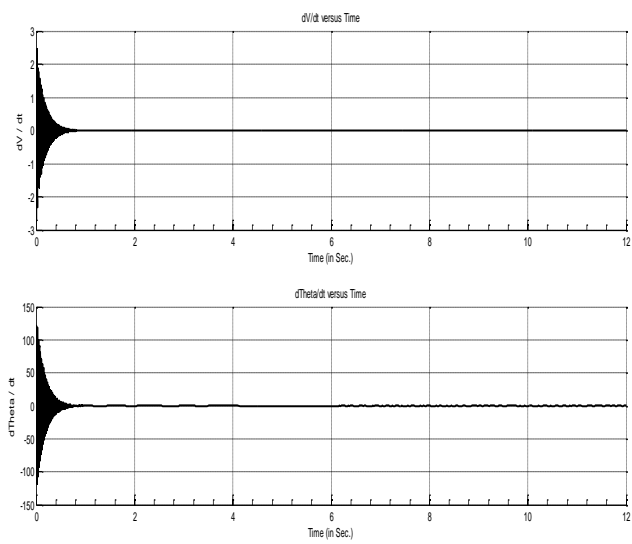

Fig. 40. $\mathrm{dV} / \mathrm{dt}$ and $\mathrm{d} \theta / \mathrm{dt}$ versus time of the $30 \mathrm{kVA}$ wind generation unit for normal operation status.

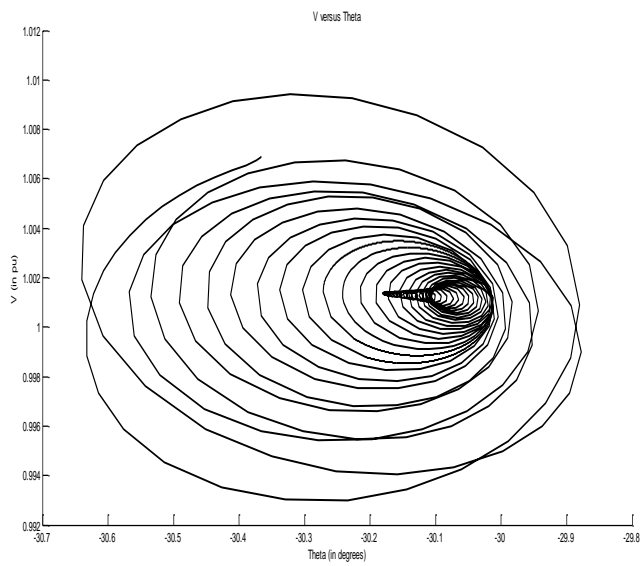

Fig. 39. V versus $\theta$ of the $30 \mathrm{kVA}$ wind generation unit for normal operation status.

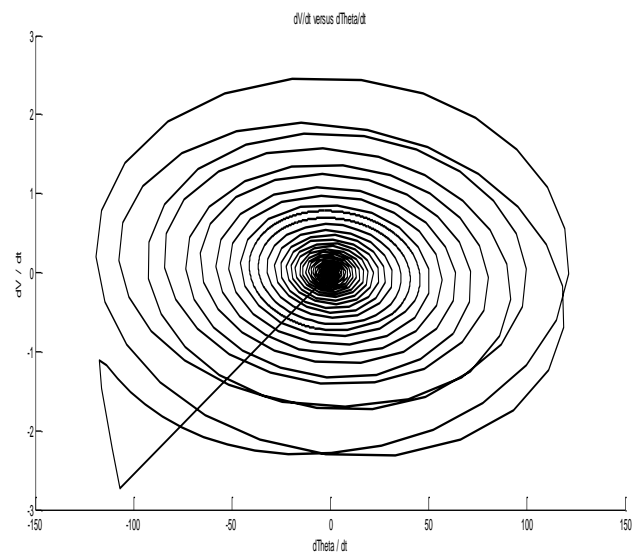

Fig. 41. $d V / d t$ versus $d \theta / d t$ of the $30 \mathrm{kVA}$ wind generation unit for normal operation status.

\section{B. Load Disconnection Status:}

The graphical results of this case have close behaviors to that of the last studied cases except the high density spiral shape of $(\mathrm{V}-\theta)$. It also differs in that the two shifted spiral shapes are nested as presented in Fig. 43 . 

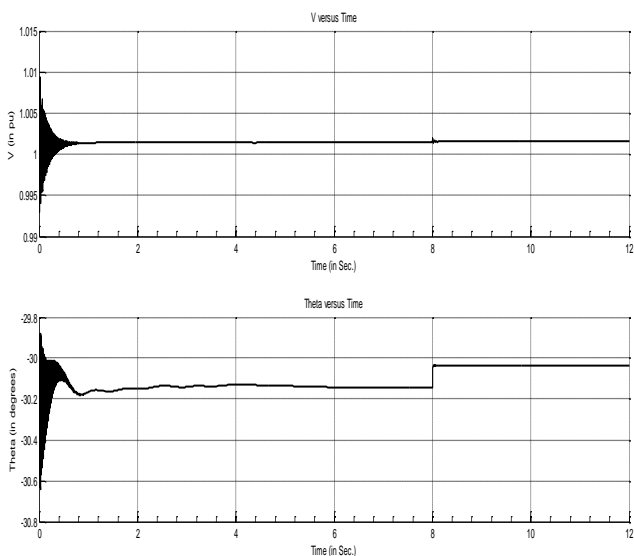

Fig. 42. V and $\theta$ versus time of the $30 \mathrm{kVA}$ wind generation unit for load disconnection status.
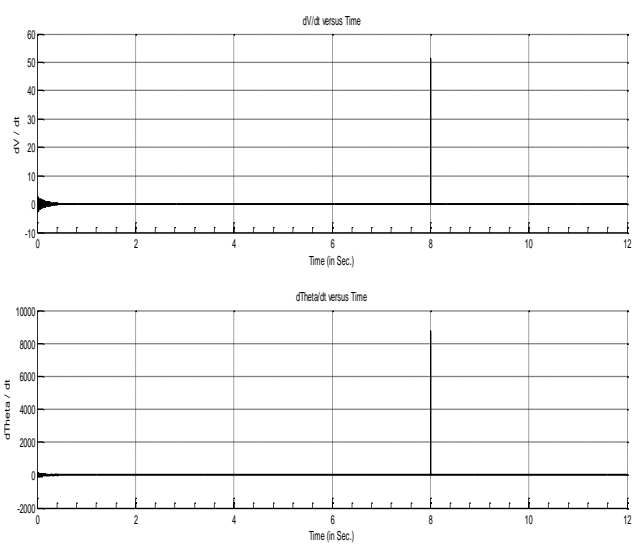

Fig. 44. $\mathrm{dV} / \mathrm{dt}$ and $\mathrm{d} \theta / \mathrm{dt}$ versus time of the $30 \mathrm{kVA}$ wind generation unit for load disconnection status.

\section{Islanding Status:}

For the islanding status, the (V - $\theta$ ) graph, shown in Fig. 47, indicates an oscillatory bi-directional spike. It exists from the right side of a spiral shape, at the time of the islanding. The relationship between $\mathrm{dV} / \mathrm{dt}$ and $\mathrm{d} \theta / \mathrm{dt}$ differs completely from that of the medium and high wind electrical power capacities studied systems as clarified in Fig. 49. It starts with a high density small spiral shape around $(0,0)$ point, then falls in form of a bidirectional positive slope sharp spike.
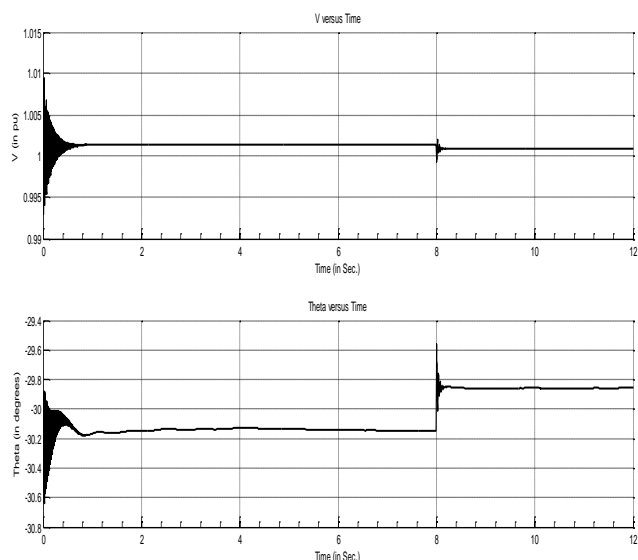

Fig. 46. $\mathrm{V}$ and $\theta$ versus time of the $30 \mathrm{kVA}$ wind generation unit for islanding status.

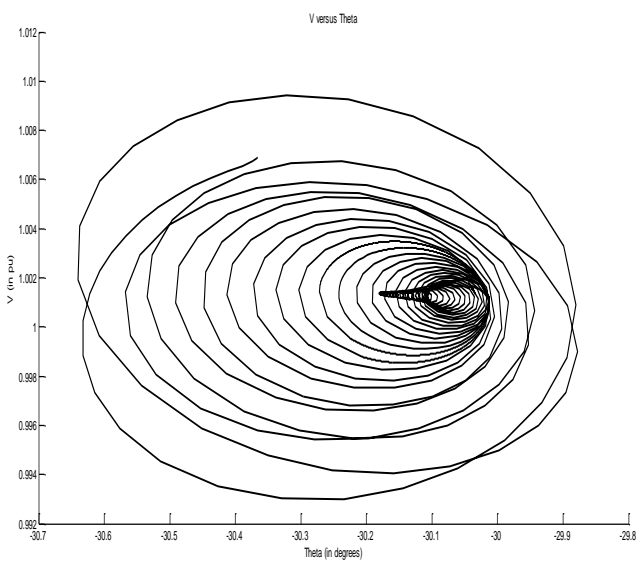

Fig. 43. V versus $\theta$ of the $30 \mathrm{kVA}$ wind generation unit for load disconnection status.

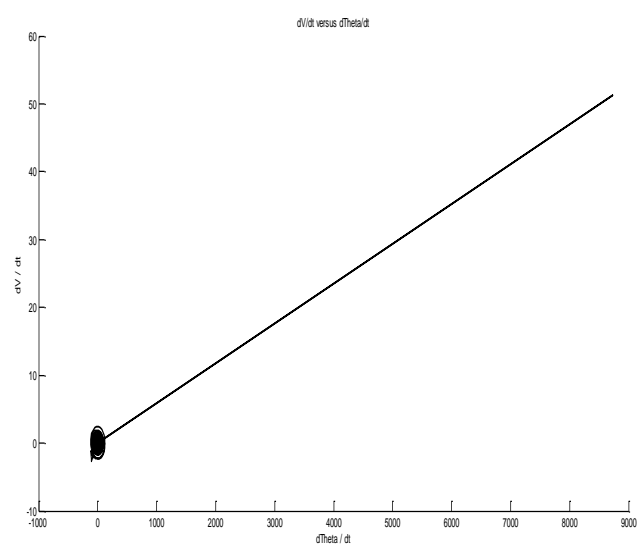

Fig. 45. $d V / d t$ versus $d \theta / d t$ of the $30 \mathrm{kVA}$ wind generation unit for load disconnection status. 

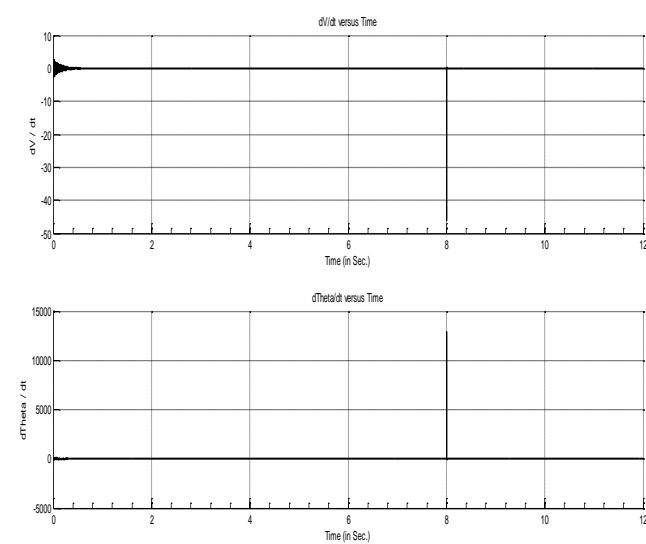

Fig. 48. $\mathrm{dV} / \mathrm{dt}$ and $\mathrm{d} \theta / \mathrm{dt}$ versus time of the $30 \mathrm{kVA}$ wind generation unit for islanding status.

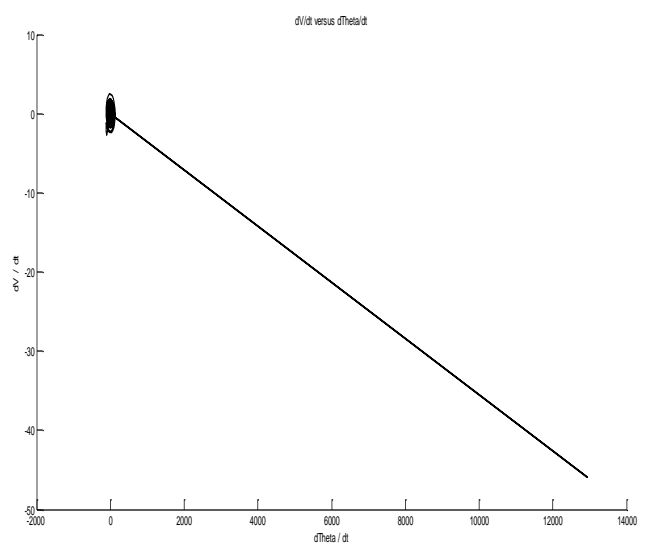

Fig. 49. $d V / d t$ versus $d \theta / d t$ of the $30 \mathrm{kVA}$ wind generation unit for islanding status.

D. Three Phase Fault Status:

The $(\mathrm{V}-\theta)$ graph is presented in Fig. 51. It can be observed that $(\mathrm{V})$ drops, on a positive slope line, to oscillate around $\mathrm{V}=0$, after fault occurrence. An oscillatory straight line around $\mathrm{dV} / \mathrm{dt}=0$, with a positive slope spike which comes from its left side, describes the $(\mathrm{dV} / \mathrm{dt}-\mathrm{d} \theta / \mathrm{dt}$ ) relationship, as illustrated in Fig. 53.
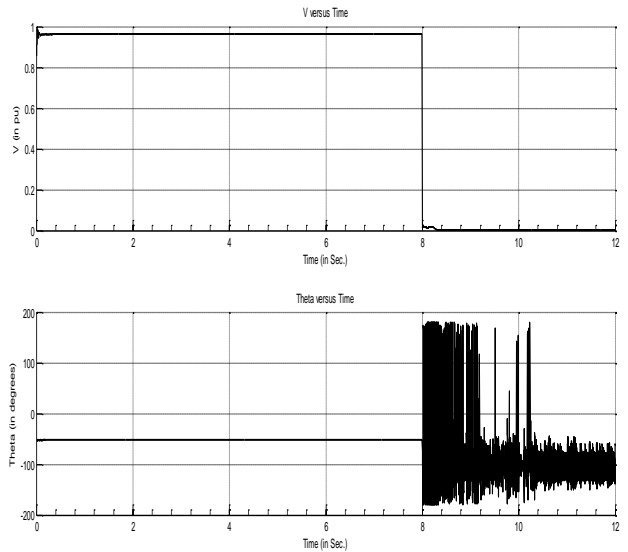

Fig. 50. V and $\theta$ versus time of the $30 \mathrm{kVA}$ wind generation unit for three phase fault status.
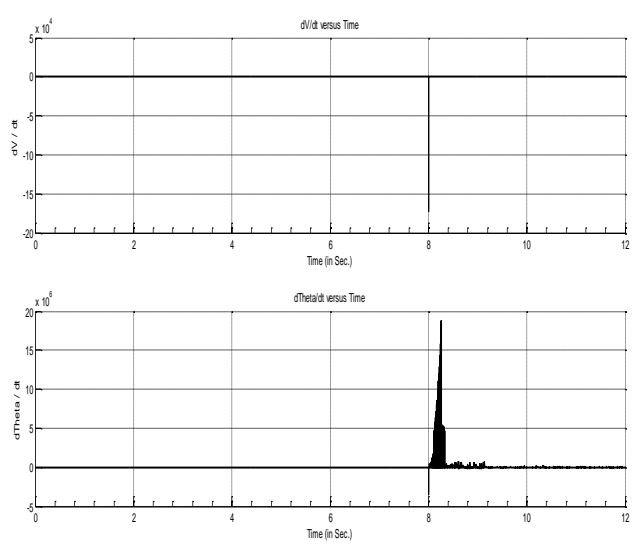

Fig. 52. $\mathrm{dV} / \mathrm{dt}$ and $\mathrm{d} \theta / \mathrm{dt}$ versus time of the $30 \mathrm{kVA}$ wind generation unit for three phase fault status.

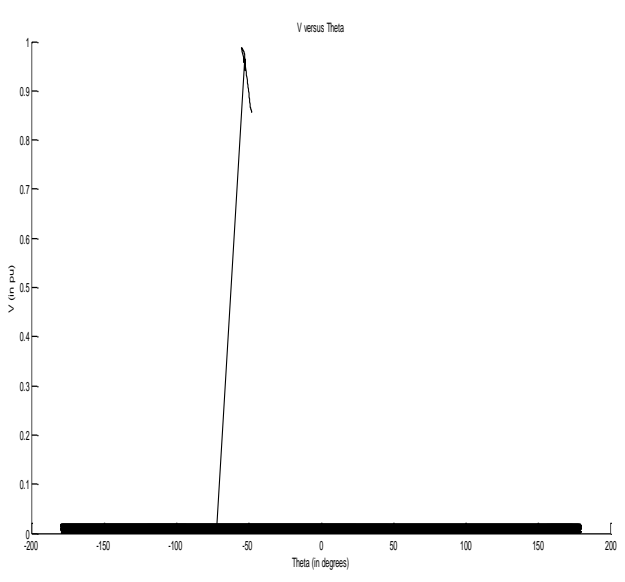

Fig. 51. V versus $\theta$ of the $30 \mathrm{kVA}$ wind generation unit for three phase fault status.

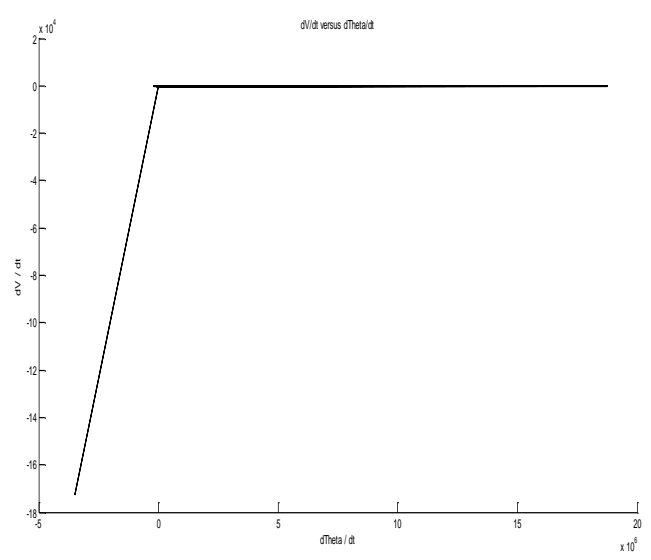

Fig. 53. $d V / d t$ versus $d \theta / d t$ of the $30 \mathrm{kVA}$ wind generation unit for three phase fault status. 


\section{Forth Case:}

\section{High Power Capacity Wind Electrical Power Generation Unit}

The power capacity of the wind electrical power generation unit is $12 \mathrm{MVA}$ in this case. It is connected to a dynamic load of $5.8 \mathrm{MW}$ and $900 \mathrm{kVAR}$ parallel with a static (parallel R-L-C) load of $6 \mathrm{MW}, 1 \mathrm{kVAR}$ inductive and $1 \mathrm{kVAR}$ capacitive.

This system has graphical results for normal operation, load disconnection, islanding, and three phase fault statuses similar to the results of the medium power capacity wind electrical power generation units in the first and second studied power systems. These graphical results are clarified in Fig. 54 to Fig. 69.

\section{A. Normal Operation Status:}
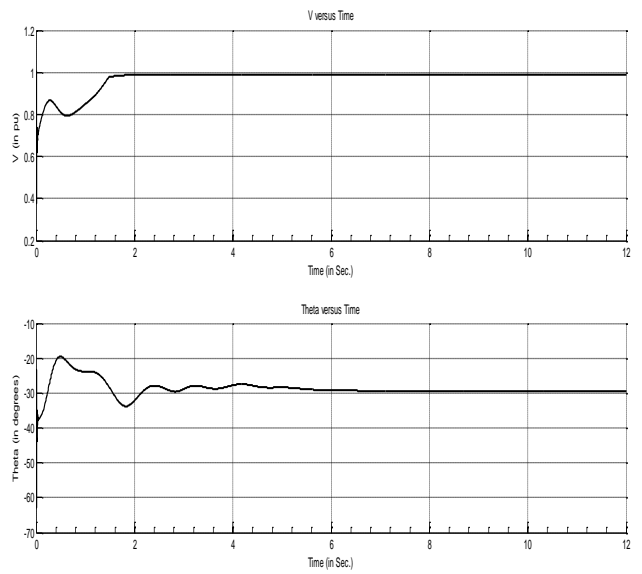

Fig. 54. $\mathrm{V}$ and $\theta$ versus time of the $12 \mathrm{MVA}$ wind generation unit for normal operation status.
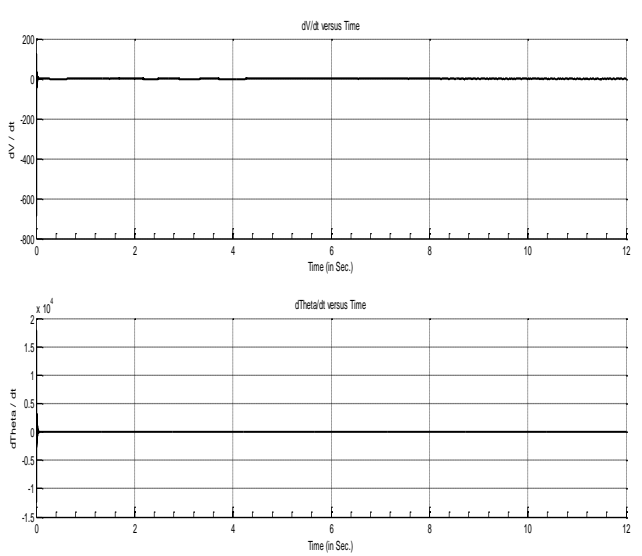

Fig. 56. $d V / d t$ and $d \theta / d t$ versus time of the $12 \mathrm{MVA}$ wind generation unit for normal operation status.

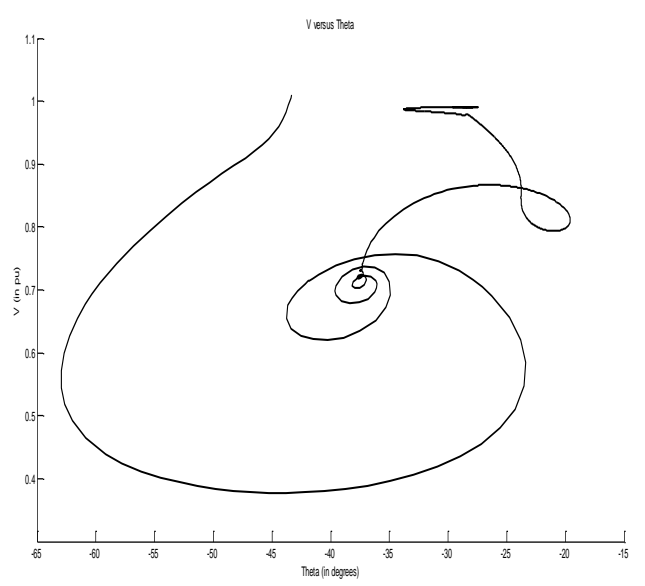

Fig. 55. V versus $\theta$ of the 12 MVA wind generation unit for normal operation status.

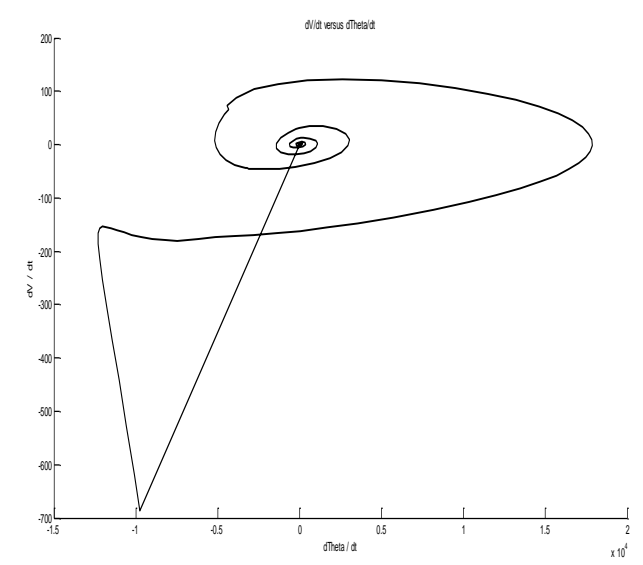

Fig. 57. $d V / d t$ versus $d \theta / d t$ of the 12 MVA wind generation unit for normal operation status.

\section{B. Load Disconnection Status:}



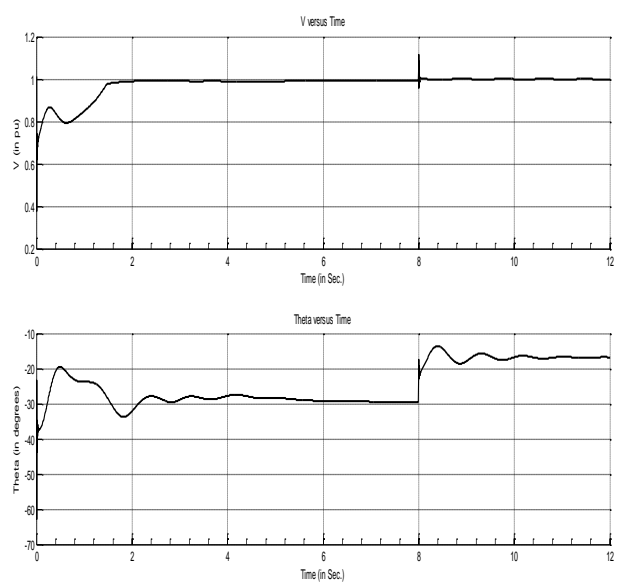

Fig. 58. $\mathrm{V}$ and $\theta$ versus time of the $12 \mathrm{MVA}$ wind generation unit for load disconnection status.
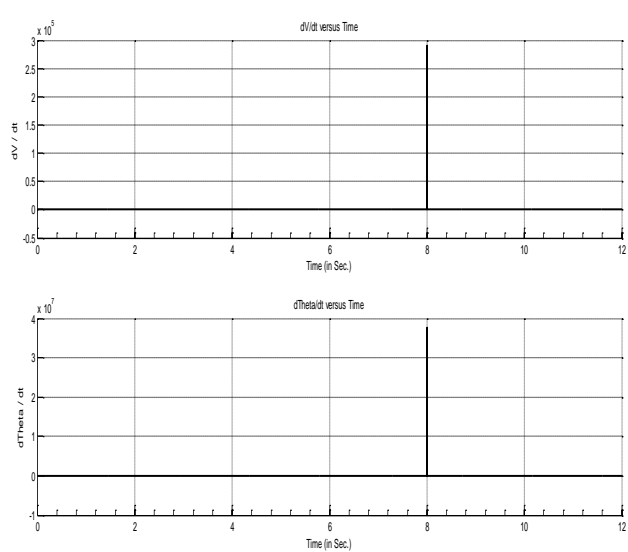

Fig. 60. $d V / d t$ and $d \theta / d t$ versus time of the $12 \mathrm{MVA}$ wind generation unit for load disconnection status.

\section{Islanding Status:}
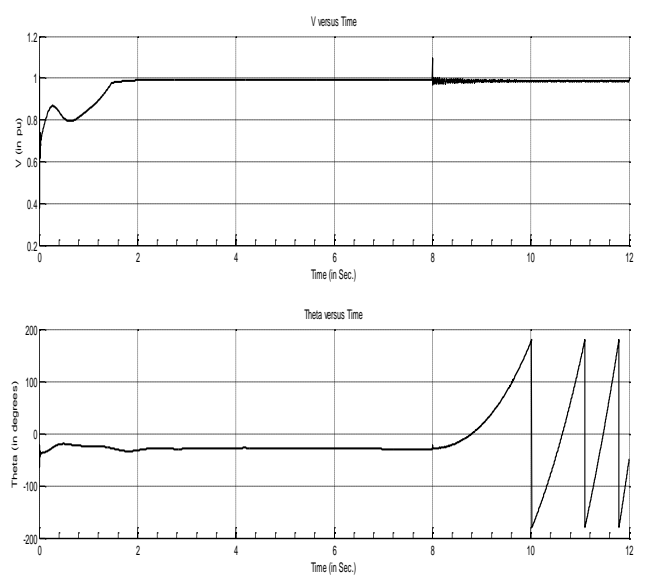

Fig. 62. V and $\theta$ versus time of the 12 MVA wind generation unit for islanding status.

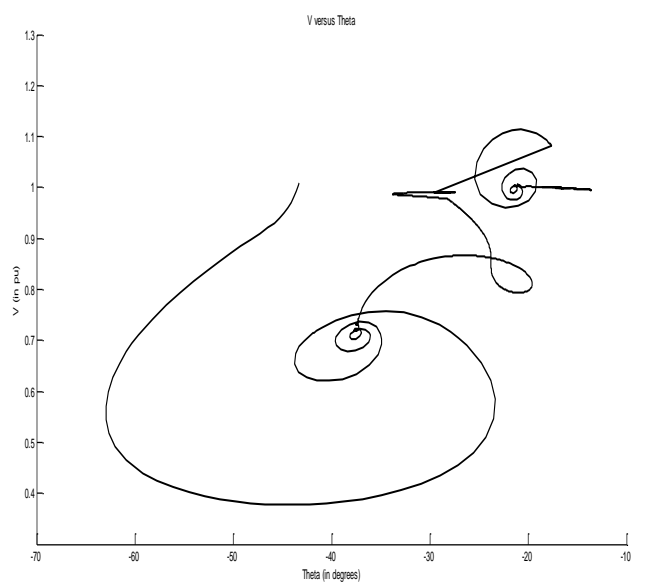

Fig. 59. V versus $\theta$ of the 12 MVA wind generation unit for load disconnection status.

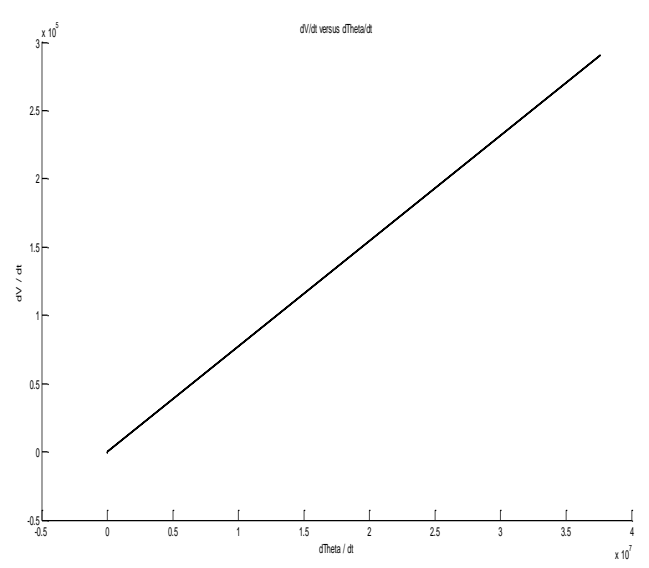

Fig. 61. $d V / d t$ versus $d \theta / d t$ of the 12 MVA wind generation unit for load disconnection status.

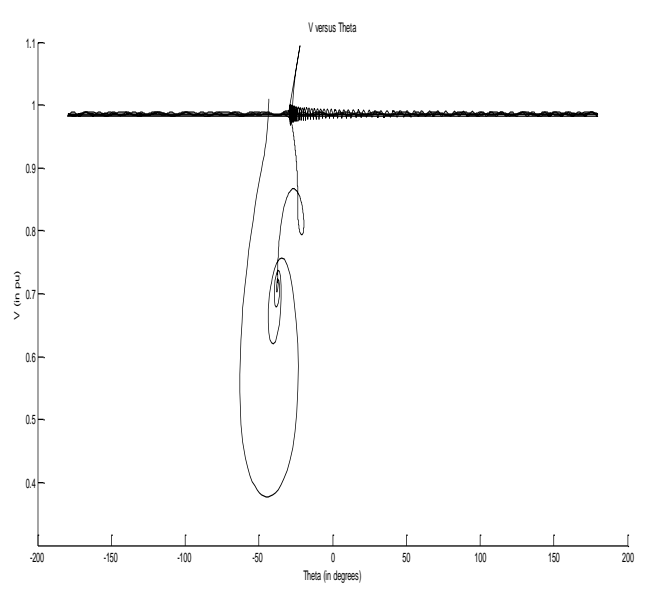

Fig. 63. V versus $\theta$ of the $12 \mathrm{MVA}$ wind generation unit for islanding status. 

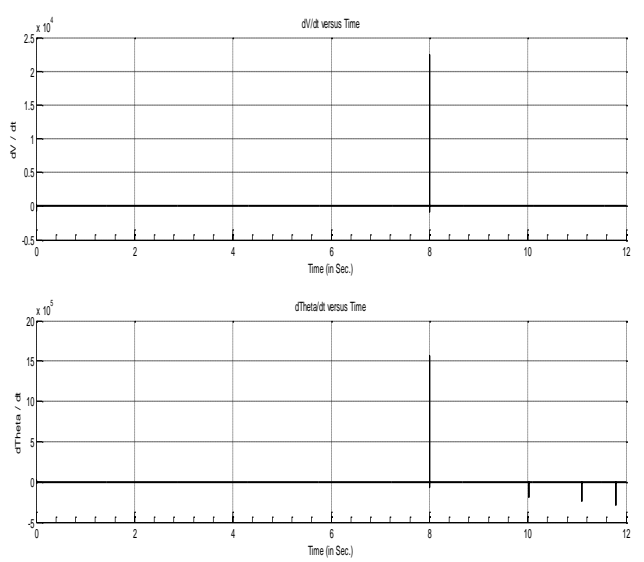

Fig. 64. $d V / d t$ and $d \theta / d t$ versus time of the 12 MVA wind generation unit for islanding status.

\section{Three Phase Fault Status:}
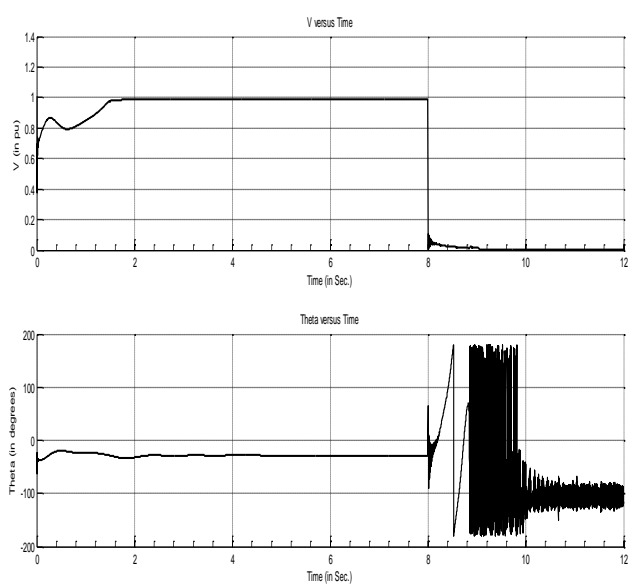

Fig. 66. $\mathrm{V}$ and $\theta$ versus time of the $12 \mathrm{MVA}$ wind generation unit for three phase fault status.
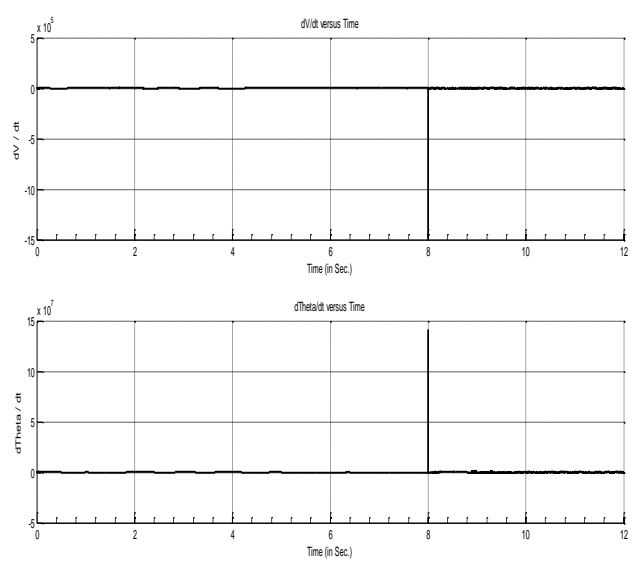

Fig. 68. $d V / d t$ and $d \theta / d t$ versus time of the 12 MVA wind generation unit for three phase fault status.

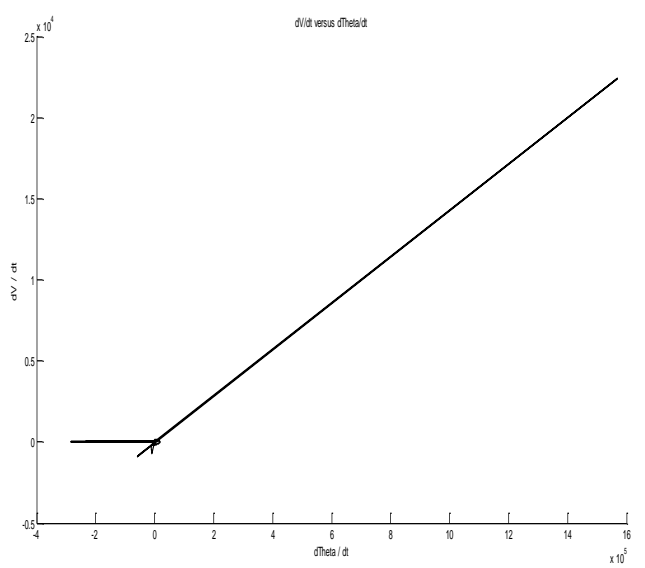

Fig. 65. $d V / d t$ versus $d \theta / d t$ of the 12 MVA wind generation unit for islanding status.

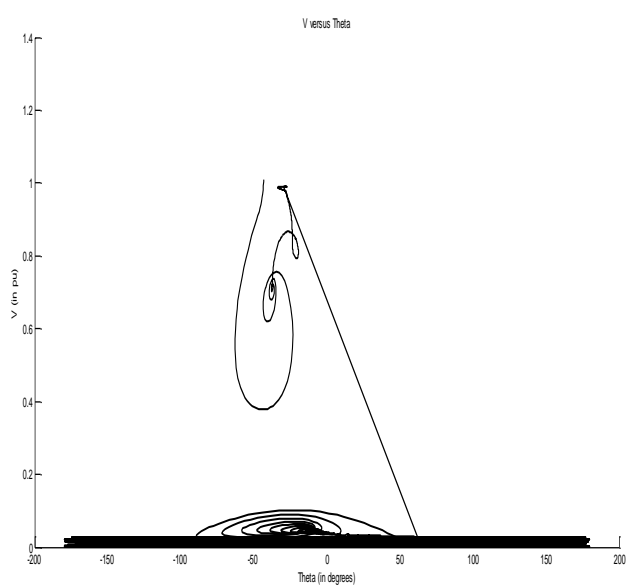

Fig. 67. V versus $\theta$ of the 12 MVA wind generation unit for three phase fault status.

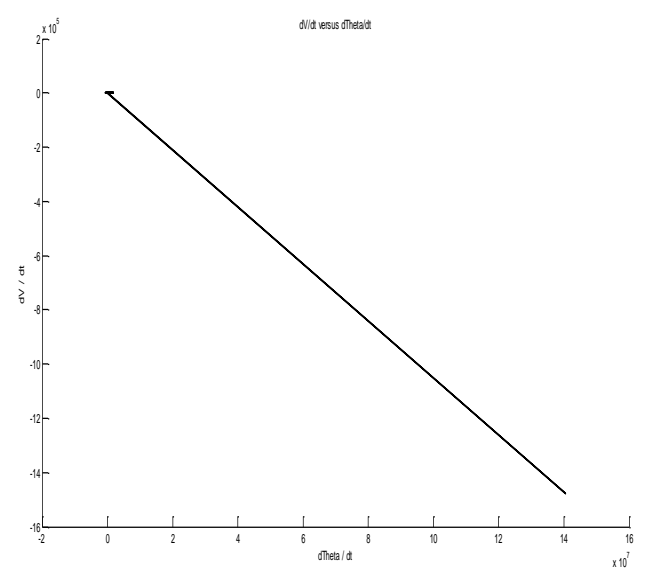

Fig. 69. $d V / d t$ versus $d \theta / d t$ of the 12 MVA wind generation unit for three phase fault status.

\section{CONCLUSION}

A new smart passive graphical islanding detection method is discussed in this paper. The proposed islanding method targets to integrate the advantages of all islanding techniques and avoid their disadvantages. 
The idea of the smart islanding method is the observation of the instantaneous phase voltage magnitude (V) variation with respect to the variation in the instantaneous phase voltage angle $(\theta)$. The variation of the voltage angle presents an accurate mirror to the different types of system transients. The behavior of the angle variation differs according to the cause of the system disturbance. Phasor measurement units (PMU) and the continuous developments in their technology realize the idea of measuring and tracing the voltage angle variation.

In this research, the $(V-\theta)$ graphs and the graphs of their derivatives $(d V / d t-d \theta / d t)$, of the bus which connects the suggested island with the main power grid, are analyzed and studied. They clarify the transient status of the power system. The new proposed smart method is applied to four different studied power systems. The four studied systems is designed of a main power grid which is connected to a wind electrical power generation unit. The main grid is similar in the four studied system, while the power generation and loading capacities of the wind electrical power generation unit differs in each studied case. The studied cases are classified according to the wind electrical power generation capacity into low, high, medium (with load-A), and medium (with load-B). The system normal operation, load disconnection, islanding, and three phase fault conditions are studied for each power system. By comparing the results of the four operating conditions for each studied system, it is obviously clear the different graphical behaviors. The $(V-\theta)$ and $(d V / d t-d \theta / d t)$ graphs of the high and medium power capacity suggested island cases (the first, second and forth studied systems) seem to be alike for similar operating conditions. The low power capacity suggested island case (the third studied system) has slightly different $(\mathrm{V}-\theta)$ and $(\mathrm{dV} / \mathrm{dt}-\mathrm{d} \theta / \mathrm{dt})$ graphs.

In the normal operation, $(\mathrm{V}-\theta)$ graph is a spiral shape around one nucleus with a steady state operating point out of this nucleus. The $(\mathrm{dV} / \mathrm{dt}-\mathrm{d} \theta / \mathrm{dt})$ graph is also a spiral but closed continuous spiral shape.

In the load disconnection condition, ( $\mathrm{V}-\theta$ ) graph has two spiral connected shapes around two different nearby nuclei. The first spiral shape represents the operation before disconnecting the load. After load disconnection, the system operates around another shifted nucleus. The $(\mathrm{dV} / \mathrm{dt}-\mathrm{d} \theta / \mathrm{dt})$ graph has a very tiny spiral shape, which seems as a point, with a long spike. The spike has positive slope with the tiny spiral shape in its bottom edge.

In the islanding condition, $(\mathrm{V}-\theta)$ graph has a spiral form with an oscillatory line shape. The oscillatory line shape is long and at the top of the spiral form, where $\mathrm{V}$ is nearly one, in the high and medium power capacity island cases, while it is short and at the right side of the spiral form in the low power capacity island case. In the high and medium power capacity island cases, the (dV/dt - d $d / d t)$ graphs show a broken line with an obtuse angle, which faces the y-axis. In the low power capacity island case, the (dV/dt - d $/$ /dt) graph has a small spiral shape, with a negative slope long spike.

In the three phase fault condition, $(\mathrm{V}-\theta)$ graph has a spiral form which falls down, after fault occurrence, to continue oscillating at $\mathrm{V}=0$. In the low power capacity suggested island case, the spiral form is very tiny. In the high and medium power capacity suggested island cases, the (dV/dt - d $0 / \mathrm{dt}$ ) graphs show a negative slope long spike with a very small spiral oscillatory shape at its top edge. On the other hand, a positive slope long spike with an oscillatory line shape at its top edge is illustrated in the low power capacity suggested island case.

The new smart graphical islanding method is a fast, accurate and inexpensive method which doesn't affect the system behavior.

\section{REFERENCES}

[1] Wei Yee Teoh, and Chee Wei Tan, "An Overview of Islanding Detection Methods in Photovoltaic Systems", World Academy of Science, Engineering and Technology, WASET, Vol. 58, pp. 577-585, 2011.

[2] A. Etxegarai, P. Eguía, and I. Zamora, "Analysis of Remote Islanding Detection Methods for Distributed Resources", Proceeding of International Conference on Renewable Energies and Power Quality, ICREPQ' 11, Las Palmas de Gran Canaria, Spain, $13^{\text {th }}-15^{\text {th }}$ April, 2011.

[3] Chandra Shekhar Chandrakar, Bharti Dewani, and Deepali Chandrakar, "An Assessment of Distributed Generation Islanding Detection Methods", International Journal of Advances in Engineering \& Technology, IJAET, Vol. 5, Issue 1, pp. 218-226, November, 2012.

[4] Asish Ranjan, S. Prabhakar Karthikeyan, et. al, "Impact of Reactive Power in Power Evacuation from Wind Turbines", Journal of Electromagnetic Analysis \& Applications, Issue No. 1, pp. 15-23, 2009. (Published online March 2009 in SciRes, internet website: www.SciRP.org/journal/jemaa).

[5] Xiaolin Ding, Peter A. Crossley, and D. John Morrow, "Islanding Detection for Distributed Generation", Journal of Electrical Engineering \& Technology, Vol. 2, No. 1, pp. 19-28, March 2007.

[6] N. Farhan, A. Rajesh, and V Y. Mohammad, "Unintentional Islanding and Comparison of Prevention Techniques", Proceedings of 37th Annual North American Conference, pp. 90-96, 2005.

[7] Noha H. El-Amary, and S. ElSafty, "Early Detection of Voltage Instability in Distribution System utilizing Phasor Measurement Units", Proceeding of International Conference on Renewable Energies and Power Quality, ICREPQ'10, Granada, Spain, $2^{3 \text { th }}-2^{5 \text { th }}$ March, 2010.

[8] A. G. Phadke, "Synchronized Phasor Measurements- A Historical Overview", IEEE Transmission and Distribution Conference and Exhibition, pp. 476-479, 2002.

internet website: http://www.phasor-rtdms.com/phaserconcepts/phasor_adv_faq.html \#Question7 (checked on 31st Dec., 2013). 